\title{
DISCOVERY OF 11 NEW T DWARFS IN THE TWO MICRON ALL SKY SURVEY, INCLUDING A POSSIBLE L/T TRANSITION BINARY
}

\author{
DAGNY L. LOOPER ${ }^{1}$ \\ Institute for Astronomy, University of Hawai'i, 2680 Woodlawn Drive, Honolulu, HI 96822, USA; dagny@ifa.hawaii.edu
}

J. DaVY KirKPATRICK ${ }^{1}$

Infrared Processing and Analysis Center, MS 100-22, California Institute of Technology, Pasadena, CA 91125, USA

AND

ADAM J. BURGASSER ${ }^{1}$

MIT Kavli Institute for Astrophysics and Space Research, 77 Massachusetts Avenue, Building 37-664B, Cambridge, MA 02139, USA Received 2007 March 28; accepted 2007 May 30

\begin{abstract}
We present the discovery of 11 new $\mathrm{T}$ dwarfs, found during the course of a photometric survey for mid-to-late $\mathrm{T}$ dwarfs in the 2MASS Point Source Catalog and from a proper-motion-selected sample of ultracool dwarfs in the 2MASS Working Database. Using the NASA Infrared Telescope Facility SpeX spectrograph, we obtained lowresolution $(R \sim 150)$ spectroscopy, allowing us to derive near-infrared spectral types of T2-T8. We also present improved signal-to-noise ratio SpeX low-resolution spectroscopy of the near-infrared T0 standard SDSS J120747.17+ 024424.8 and T1 standard SDSSp J083717.22-000018.3. One of these new T dwarfs, 2MASS J13243559+ 6358284 , was also discovered independently by Metchev et al. This object is spectroscopically peculiar and possibly a binary and/or very young $(<300 \mathrm{Myr})$. We specifically attempted to model the spectrum of this source as a composite binary to reproduce its peculiar spectral characteristics. The latest type object in our sample is a T8 dwarf, 2MASS J07290002-3954043, now one of the four latest type T dwarfs known. All $11 \mathrm{~T}$ dwarfs are nearby given their spectrophotometric distance estimates, with one $\mathrm{T}$ dwarf within $10 \mathrm{pc}$ and eight additional $\mathrm{T}$ dwarfs within $25 \mathrm{pc}$, if single. These new additions increase the $25 \mathrm{pc}$ census of $\mathrm{T}$ dwarfs by $\sim 14 \%$. Their proximity offers an excellent opportunity to probe for companions at separations closer than are possible for more distant $\mathrm{T}$ dwarfs.
\end{abstract}

Key words: stars: low-mass, brown dwarfs — techniques: spectroscopic

Online material: color figure

\section{INTRODUCTION}

The T spectral class (Burgasser et al. 2002; Geballe et al. 2002) is currently the coolest populated class on the MK system, with effective temperatures ranging from $\sim 1400 \mathrm{~K}$ at the $\mathrm{L} / \mathrm{T}$ transition down to $\sim 700 \mathrm{~K}$ for the coolest known T dwarf (Golimowski et al. 2004; Vrba et al. 2004; Kirkpatrick 2005). The transition between the $\mathrm{L}$ and $\mathrm{T}$ spectral classes is characterized by the onset of $\mathrm{CH}_{4}$ absorption in the near-infrared (NIR). By mid-type $\mathrm{T}$, the spectral class is dominated by $\mathrm{CH}_{4}, \mathrm{H}_{2} \mathrm{O}$, and collision-induced absorption by $\mathrm{H}_{2}\left(\mathrm{CIA} \mathrm{H}_{2}\right.$ ) in the NIR (Saumon et al. 1994; Borysow et al. 1997; Kirkpatrick et al. 2006).

Since the discovery of the T prototype Gl 229B (Nakajima et al. 1995; Oppenheimer et al. 1995) in 1995, 100 T dwarfs have been identified. ${ }^{2}$ These objects are our coolest neighbors, spanning the mass and temperature gap between giant planets and the lowest mass stars. Unlike extrasolar planets, we can directly image and often spectroscopically observe $\mathrm{T}$ dwarfs in the field and as companions to more massive stars. Therefore, these objects offer invaluable testbeds for extrasolar giant planet atmospheric models.

Due to their intrinsic faintness, the census of $\mathrm{T}$ dwarfs in the solar neighborhood remains largely incomplete. To date, two surveys have provided the largest contributions to the currently known population: the Sloan Digitized Sky Survey (SDSS; York

\footnotetext{
${ }^{1}$ Visiting Astronomer at the Infrared Telescope Facility, which is operated by the University of Hawaii under Cooperative Agreement NCC 5-538 with the National Aeronautics and Space Administration, Science Mission Directorate, Planetary Astronomy Program.

${ }^{2}$ See http://dwarfarchives.org for a full list of $\mathrm{T}$ dwarfs.
}

et al. 2000), a wide-field optical survey, and the Two Micron All Sky Survey (2MASS; Skrutskie et al. 2006), a NIR all sky survey. $\mathrm{T}$ dwarfs emit most of their flux in the NIR; however, due to the $J-K_{s}$ color degeneracy of early-type $\mathrm{T}$ dwarfs and $\mathrm{M}$ dwarfs, recovery of early-type T dwarfs by the 2MASS survey has proven difficult. The SDSS survey, while successful at identifying earlytype (hotter) T dwarfs using far-optical color selections, is not as sensitive to late-type (cooler) $\mathrm{T}$ dwarfs, which emit very little flux at visual wavelengths.

To expand the census of the Sun's coolest neighbors, we have conducted a photometric survey for mid-to-late-type $\mathrm{T}$ dwarfs using the 2MASS Point Source Catalog, searching both deeper than previous surveys for T dwarfs (Burgasser et al. 1999, 2000a, 2000b, 2002, 2003a, 2003c, 2004b; Tinney et al. 2005; Ellis et al. 2005 ) and in the Galactic plane. We present the discovery of eight new $\mathrm{T}$ dwarfs from this search, including the discovery of a very nearby $(d \sim 8.4 \mathrm{pc}) \mathrm{T} 8 \mathrm{dwarf}$. We also present the first three $\mathrm{T}$ dwarfs from our 2MASS proper-motion search, all of which are early-type $T$ dwarfs. We describe these two searches in $\S 2$. In $\S 3$ we describe the spectroscopic observations obtained with the NASA Infrared Telescope Facility $3.0 \mathrm{~m}$ SpeX spectrograph and the characterization of this new set of $T$ dwarfs. In $\S 4$ we discuss the current census of the solar neighborhood. Finally, in $\S 5$ we give our conclusions.

\section{OBSERVATIONS}

\subsection{Target Selection}

The 11 new T dwarfs we report here were discovered in two different searches using Two Micron All Sky Survey (2MASS) 
TABLE 1

Description of 2MASS Photometric Selection of T Dwarfs

\begin{tabular}{|c|c|c|c|c|c|c|c|c|}
\hline \multirow[b]{2}{*}{ Galactic Cut } & \multirow[b]{2}{*}{$J$ Magnitude Cut } & \multirow[b]{2}{*}{ Color Cuts } & \multicolumn{2}{|c|}{ No. of CANDidates } & \multirow[b]{2}{*}{ FOLLOWED-UP } & \multirow[b]{2}{*}{ Transients } & \multirow[b]{2}{*}{ M DWARFS } & \multirow[b]{2}{*}{ T Dwarfs } \\
\hline & & & Cut $1^{\text {a }}$ & Cut $2^{\mathrm{b}}$ & & & & \\
\hline$|b| \geq 15^{\circ}$ & $16<J \leq 16.5^{\mathrm{c}}$ & $\begin{array}{l}(J-H \leq 0) \text { or } \\
\left(J-H \leq 0.3, H-K_{s} \leq 0\right)\end{array}$ & 13233 & $67(+3)^{d}$ & 56 & 44 & 8 & $4(+3)$ \\
\hline $10^{\circ} \leq|b|<15^{\circ}$ & $J \leq 16.5$ & $\begin{array}{l}(J-H \leq 0) \text { or } \\
\left(J-H \leq 0.3, H-K_{s} \leq 0\right)\end{array}$ & 2719 & 8 & 6 & 4 & 1 & 1 \\
\hline$|b| \leq 10^{\circ},|l| \geq 20^{\circ} \ldots \ldots \ldots \ldots \ldots$ & $J \leq 16$ & $J-H \leq 0$ & 3711 & 6 & 5 & 2 & 0 & 3 \\
\hline
\end{tabular}

data: (1) a 2 MASS photometric search for mid-to-late-type T dwarfs and (2) a 2MASS proper-motion survey for ultracool dwarfs.

\subsubsection{MASS Photometric Selection of Mid-to-Late Type T Dwarfs}

This search was designed to identify mid-to-late-type T dwarfs and to be complementary to previous searches by Burgasser et al. using the 2MASS database. Their most recent search selected sources with $|b| \geq 15^{\circ}, J \leq 16.0, J-H \leq 0.3$, or $H-K_{s} \leq 0$, and no optical counterpart within a $5^{\prime \prime}$ radius in the USNO-A2.0 catalog or by visual inspection of DSS I and II images. We extended our search half a magnitude deeper in the $J$ band for the same area of sky $\left(|b| \geq 15^{\circ}, 16<J \leq 16.5\right)$ and searched the Galactic plane $\left(|b|<15^{\circ}\right)$, a portion of sky completely unexplored by Burgasser et al. We also modified our color selection criteria, described below, to decrease the number of false positives followed-up spectroscopically.

Our search was broken into three parts defined by Galactic latitude: (1) $|b| \geq 15^{\circ}$, (2) $10^{\circ} \leq|b|<15^{\circ}$, and (3) $|b|<10^{\circ}$. All three searches had the following keywords (in parentheses) in common:

1. Not a cataloged minor planet at the time the 2MASS Point Source Catalog was constructed $\left(\mathrm{mp} \_\right.$flg $\left.=0\right)$.

2. No contamination by galaxies (gal_contam $=0$ ).

3. No artifact contamination or source confusion (cc_flg like "000").

4. No optical counterpart within a $5^{\prime \prime}$ radius in the USNO-A2.0 Catalog (nopt_mchs $=0$ ) or by visual inspection of DSS II $I$-band images. ${ }^{3}$

5. A non-null detection in both the $J$ and $H$ bands ( $\mathrm{j}_{-} \mathrm{cmsig}$ is not null and $\mathrm{h}$ _cmsig is not null).

6. Sky positions not coincident with the Large Magellanic Cloud, Small Magellanic Cloud, or 47 Tuc.

The $J$-band magnitude and NIR color cuts for each of the three Galactic latitude searches are defined in Table 1. For (1) $|b| \geq 15^{\circ}$ and (2) $10^{\circ} \leq|b|<15^{\circ}$, we used the same color selection $\left[(J-H \leq 0)\right.$ or $\left(J-H \leq 0.3\right.$ and $\left.\left.H-K_{s} \leq 0\right)\right]$. This color selection is similar to but more restrictive than that used by Burgasser et al. (2003a; $J-H \leq 0$ or $H-K_{s} \leq 0$ ) and was modified from that search because of the high incidence of M dwarfs ( $~ 89 \%)$ and low incidence of $\mathrm{T}$ dwarfs in spectroscopic follow-up ( 11\%; Burgasser et al. 2004b). Burgasser et al. had relaxed color selection criteria to allow detection of early-type T dwarfs (<T4). For (3) $|b|<10^{\circ}$, we further restricted our color

\footnotetext{
${ }^{3}$ Images acquired from http://cadcwww.dao.nrc.ca/cadcbin/getdss.
}

selection $(J-H \leq 0)$ and brightness limit $(J \leq 16)$ due to the high density of sources in the Galactic plane and searched only outside the Galactic center, $|l| \geq 20^{\circ}$. These color selections effectively limited our search to SpT $>$ T4 (see Fig. 1).

Of the three searches, the Galactic plane search (search 3, $|b|<10^{\circ}$ and $|l| \geq 20^{\circ}$ ) had the lowest rate of transients (40\%: asteroids, spurious detections, flare events, etc.; see Table 2) and, after confirmation, the highest rate of $\mathrm{T}$ dwarfs $(100 \%)$. The three new T dwarfs from this search are 2MASS $0602+40,{ }^{4}$ 2MASS 1007-45, and 2MASS 2154+59 (see Table 3). In comparison, search $1,|b| \geq 15^{\circ}$, had a transient rate of $\sim 79 \%$ and, after confirmation, a T dwarf rate of $\sim 58 \%$, with four new T dwarfs identified: 2MASS 0510-42, 2MASS 1215-34, 2MASS 1615+13, and 2MASS 2154-10 (see Table 3). Three previously known T dwarfs were also recovered: SDSS 1630+08 ( T5.5; Chiu et al. 2006), SDSS 1758+46 (T6.5; Knapp et al. 2004), and SDSS 2124+01 (T5; Knapp et al. 2004; see Table 4). Search 2, $10^{\circ} \leq$ $|b|<15^{\circ}$, had a transient rate of $\sim 67 \%$ and, after confirmation, a T dwarf rate of $50 \%$, with one new T dwarf identified: 2MASS 0729-39 (see Table 3). The lower transient and higher T dwarf rate for the Galactic plane search arises from the better photometry $(J \leq 16)$ and more restrictive color selection $(J-H \leq 0)$ used.

The total incidence of transients in these three searches was $\sim 71 \%$. In Figure 2 we show a histogram of the fraction of transients versus ecliptic latitude. The vast majority (84\%) are located within $30^{\circ}$ of the ecliptic. The remaining $16 \%$ are likely highly inclined asteroids or image artifacts. We list all 50 transients in Table 2.

By contrast, the eight newly identified and three confirmed T dwarfs are distributed nearly evenly across ecliptic latitude ( $\beta$; see Fig. 2). For all confirmed candidates, we find a fraction of 0.45 for $\mathrm{M}$ dwarfs and 0.55 for $\mathrm{T}$ dwarfs, a five-fold improvement over Burgasser et al.'s search despite more uncertain photometry for $|b| \geq 10^{\circ}$ and $16.0<J \leq 16.5$. This increased rate of $\mathrm{T}$ dwarf detection occurred because our color selection lies further from the locus of $\mathrm{M}$ dwarfs, leading to less scattering from uncertain photometry of faint $\mathrm{M}$ dwarfs into our color selection (see Fig. 1). To date, we have completed follow-up of 67 of 70 candidates visible from IRTF $^{5}$ and have 11 candidates too northerly or southerly to be observed by IRTF. Of these remaining 14 candidates, 11 have $|\beta|>30^{\circ}$. Taking into account the

\footnotetext{
${ }^{4}$ We abbreviate all of our discoveries from "2MASS Jhhmmss[.ss] \pm ddmmss[.]s" to " 2 MASS hhmm \pm dd" in the text for ease of reading but give their full designations in Table 3.

${ }^{5}$ IRTF: $\sim-48^{\circ}<\delta \leq+69^{\circ} 56^{\prime}$ (hard limit).
} 

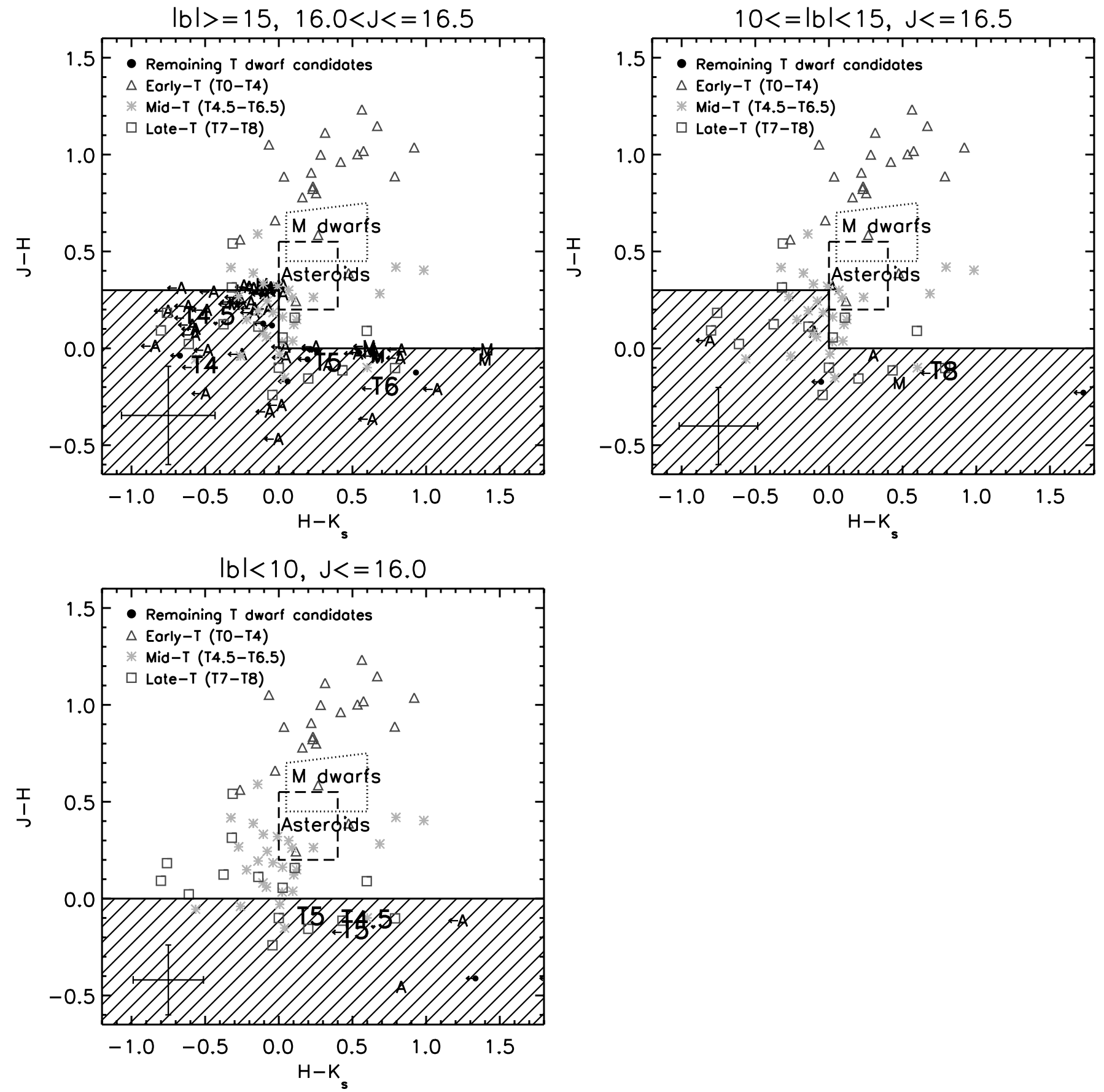

FIG. 1.-NIR color-color plots for the 2MASS photometric search for mid- to late-type T dwarfs broken down by Galactic latitude. Color selections are shown by the hatched area. Known $\mathrm{T}$ dwarfs are plotted for reference, with early-type $\mathrm{T}$ dwarfs represented by triangles, mid-type $\mathrm{T}$ dwarfs by asterisks, and late-type $\mathrm{T}$ dwarfs by squares. For reference, the loci of M dwarfs and typical asteroids are outlined (Kirkpatrick et al. [2000] and Grav \& Holman [2004], respectively). Candidates absent in follow-up imaging are denoted by "A," and spectroscopically confirmed M dwarfs are denoted by "M." All candidates needing follow-up imaging/spectroscopy are denoted by solid circles, and newly identified T dwarfs are denoted by their spectral type. For candidates with null $K_{s}$ errors, arrows indicate an upper limit on their $H-K_{s}$ color. Median error bars for all candidates with non-null color errors are shown in the lower left of each plot. [See the electronic edition of the Journal for a color version of this figure.]

photometry and ecliptic latitudes of these remaining sources, we estimate that 4-5 of these remaining candidates are T dwarfs. We have programs on IRTF SpeX, Gemini-North NIFS, and SOAR OSIRIS to obtain imaging/spectroscopy of all remaining 14 candidates and will present the results in a future paper.

\subsubsection{MASS Proper-Motion Survey}

We have carried out a proper-motion survey for ultracool dwarfs using $\sim 4700 \mathrm{deg}^{2}$ of multiepoch data in the 2MASS Working Database. We give a brief outline of the search criteria here and refer the reader to D. L. Looper et al. (2007, in preparation) for full details of this search. For this data set we selected all objects with a proper motion exceeding $0.2^{\prime \prime} \mathrm{yr}^{-1}$, a time difference between epochs of $\Delta t \geq 0.2 \mathrm{yr}$, a positional difference exceeding $0.4^{\prime \prime}$ between the first and last epochs, and $J \leq 16.5$. We also required no optical counterpart within a $5^{\prime \prime}$ radius in the USNO-A2.0 catalog (Monet et al. 1998) or by visual inspection of Digitized Sky Survey (DSS) I and II $V$ - or $R$-band images. For particularly bright objects, we required large $R-J$ colors $(R-J>6)$. This search resulted in $\sim 140$ candidates. To date, we have spectroscopically 
TABLE 2

TRANSIENTS

\begin{tabular}{|c|c|c|c|c|c|}
\hline Object $^{\mathrm{a}}$ & $J^{\mathrm{b}}$ & $H^{\mathrm{b}}$ & $K_{s}{ }^{\mathrm{b}}$ & UT Date $^{\mathrm{c}}$ & $\begin{array}{c}\beta \\
(\operatorname{deg})^{d}\end{array}$ \\
\hline 2MASS J00082060-1922267........................... & $16.26 \pm 0.09$ & $15.99 \pm 0.17$ & 16.04(null) & 1998 Aug 14 & -18.5 \\
\hline 2MASS J00511030-0704476 ........................... & $16.30 \pm 0.09$ & $16.03 \pm 0.13$ & $16.19 \pm 0.36$ & 1998 Oct 2 & -11.6 \\
\hline 2MASS J01085000-0428510 ........................... & $16.11 \pm 0.08$ & $16.23 \pm 0.19$ & $15.92 \pm 0.27$ & 1998 Sep 18 & -10.9 \\
\hline 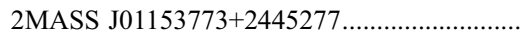 & $16.46 \pm 0.12$ & $16.16 \pm 0.22$ & 16.16(null) & 1997 Oct 31 & +15.5 \\
\hline 2MASS J01202541-0421252.. & $16.41 \pm 0.12$ & $16.25 \pm 0.25$ & 17.03(null) & 1998 Sep 20 & -11.9 \\
\hline 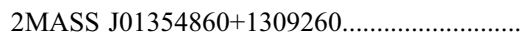 & $16.41 \pm 0.11$ & $16.44 \pm 0.23$ & 16.21(null) & 2000 Sep 24 & +3.0 \\
\hline 2MASS J02113209+1959189........................ & $16.19 \pm 0.09$ & $15.90 \pm 0.13$ & 15.99(null) & 1997 Oct 19 & +6.4 \\
\hline 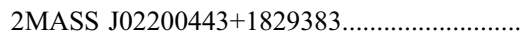 & $16.36 \pm 0.12$ & $16.07 \pm 0.17$ & $16.33 \pm 0.39$ & 1997 Oct 20 & +4.3 \\
\hline 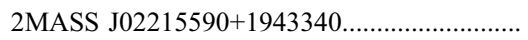 & $16.21 \pm 0.10$ & $16.26 \pm 0.22$ & $15.64 \pm 0.22$ & 1997 Oct 20 & +5.3 \\
\hline 2MASS J02242110+1926020 ............................. & $16.37 \pm 0.11$ & $16.62 \pm 0.29$ & 15.57(null) & 1997 Oct 20 & +4.8 \\
\hline 2MASS J02262810+2327310 & $16.21 \pm 0.10$ & $16.29 \pm 0.21$ & 15.49(null) & 1997 Oct 20 & +8.5 \\
\hline 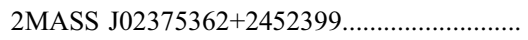 & $16.19 \pm 0.09$ & $15.93 \pm 0.15$ & $16.01 \pm 0.25$ & 1997 Nov 10 & +9.0 \\
\hline 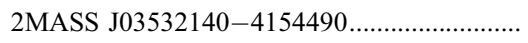 & $16.46 \pm 0.14$ & $16.85 \pm 0.36$ & 16.25(null) & 1999 Sep 17 & -59.9 \\
\hline 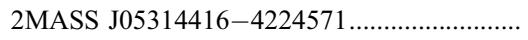 & $16.49 \pm 0.16$ & $16.85 \pm 0.36$ & 16.94(null) & 1999 Oct 29 & -65.5 \\
\hline 2MASS J06550663+3024105 .............................. & $15.89 \pm 0.08$ & $15.83 \pm 0.17$ & $15.97 \pm 0.31$ & 1998 Nov 23 & +7.5 \\
\hline 2MASS J08484742+0349184_............................ & $16.36 \pm 0.15$ & $16.40 \pm 0.28$ & 16.91(null) & 2000 Jan 29 & -13.5 \\
\hline 2MASS J09164433+0601113 ....... & $16.31 \pm 0.13$ & $16.24 \pm 0.24$ & 16.83(null) & 2000 Feb 22 & -9.3 \\
\hline 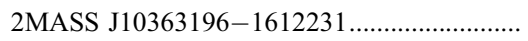 & $16.27 \pm 0.11$ & $16.03 \pm 0.15$ & 16.34(null) & 1998 Apr 2 & -23.1 \\
\hline 2MASS J11153870-0446599 …........................ & $16.42 \pm 0.10$ & $16.92 \pm 0.30$ & 16.95 (null) & 1999 Jan 4 & -8.8 \\
\hline 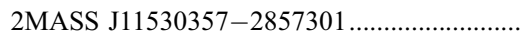 & $16.42 \pm 0.15$ & $16.74 \pm 0.36$ & 16.75(null) & $2000 \mathrm{Feb} 1$ & -27.0 \\
\hline 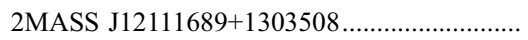 & $16.31 \pm 0.11$ & $16.05 \pm 0.18$ & 16.52(null) & 2000 Apr 5 & +13.1 \\
\hline 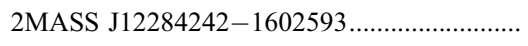 & $16.44 \pm 0.12$ & $16.28 \pm 0.19$ & 16.80(null) & 1998 Apr 1 & -11.9 \\
\hline 2MASS J12352384+2035252 & $16.14 \pm 0.10$ & $15.89 \pm 0.18$ & $15.89 \pm 0.24$ & 1999 Apr 25 & +22.3 \\
\hline 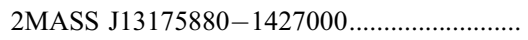 & $16.11 \pm 0.11$ & $16.15 \pm 0.21$ & 15.34(null) & 1998 Apr 6 & -5.8 \\
\hline 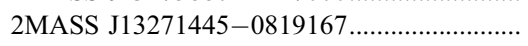 & $16.45 \pm 0.13$ & $16.51 \pm 0.31$ & 16.80 (null) & 1999 Feb 20 & +0.8 \\
\hline 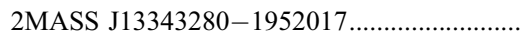 & $16.33 \pm 0.12$ & $16.08 \pm 0.17$ & 16.20 (null) & 1998 Apr 15 & -9.3 \\
\hline 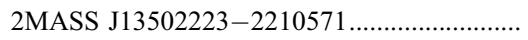 & $16.24 \pm 0.11$ & $16.03 \pm 0.23$ & 16.37 (null) & 1998 May 17 & -10.1 \\
\hline 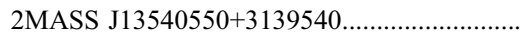 & $16.16 \pm 0.12$ & $16.19 \pm 0.28$ & 16.17(null) & 1998 Mar 11 & +40.0 \\
\hline 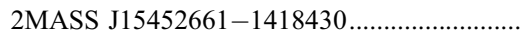 & $16.47 \pm 0.12$ & $16.29 \pm 0.20$ & 16.93(null) & 1999 Mar 21 & +5.4 \\
\hline 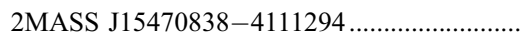 & $15.46 \pm 0.07$ & $15.17 \pm 0.08$ & $15.18 \pm 0.16$ & 1999 May 14 & -20.7 \\
\hline 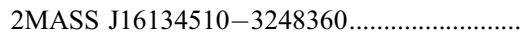 & $15.56 \pm 0.07$ & $15.63 \pm 0.14$ & $15.36 \pm 0.21$ & 1998 Jul 13 & -11.4 \\
\hline 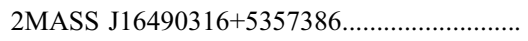 & $16.20 \pm 0.09$ & $15.91 \pm 0.16$ & 16.61(null) & 1998 Jun 14 & +74.8 \\
\hline 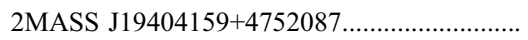 & $15.93 \pm 0.08$ & $15.92 \pm 0.14$ & 16.76(null) & 1998 Jun 26 & +67.2 \\
\hline 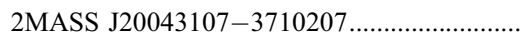 & $16.45 \pm 0.18$ & $16.72 \pm 0.48$ & 17.24(null) & 1999 Jul 4 & -16.4 \\
\hline 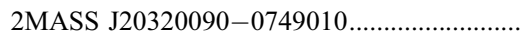 & $16.15 \pm 0.08$ & $16.22 \pm 0.16$ & 16.21(null) & 1999 Jul 6 & +10.7 \\
\hline 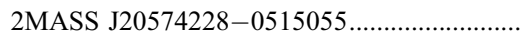 & $16.20 \pm 0.10$ & $15.92 \pm 0.21$ & 16.12(null) & 1998 Sep 20 & +11.5 \\
\hline 2MASS J21000824+4759033_.............................. & $15.79 \pm 0.08$ & $15.93 \pm 0.15$ & 14.72(null) & 2000 Jun 10 & +60.4 \\
\hline 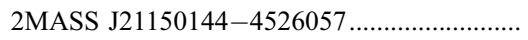 & $16.34 \pm 0.11$ & $16.36 \pm 0.26$ & 17.23(null) & 1999 Aug 13 & -28.0 \\
\hline 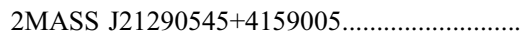 & $15.60 \pm 0.06$ & $16.09 \pm 0.20$ & $15.29 \pm 0.16$ & 2000 May 18 & +52.6 \\
\hline 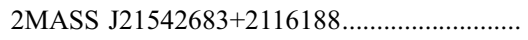 & $16.37 \pm 0.12$ & $16.22 \pm 0.24$ & 16.41(null) & 1999 Oct 2 & +31.7 \\
\hline 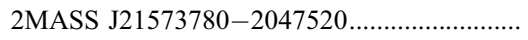 & $16.49 \pm 0.12$ & $16.55 \pm 0.24$ & 16.04(null) & 1998 Aug 11 & -7.8 \\
\hline 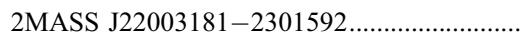 & $16.43 \pm 0.14$ & $16.34 \pm 0.28$ & 16.96(null) & 1998 Jul 4 & -10.2 \\
\hline 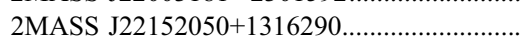 & $16.46 \pm 0.13$ & $16.25 \pm 0.19$ & 16.47 (null) & 1997 Sep 13 & +22.4 \\
\hline 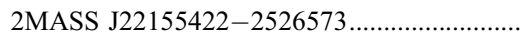 & $16.14 \pm 0.11$ & $15.85 \pm 0.14$ & 16.08(null) & 2000 Jul 24 & -13.7 \\
\hline 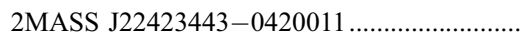 & $16.39 \pm 0.10$ & $16.18 \pm 0.18$ & 16.46(null) & 1998 Sep 29 & +3.6 \\
\hline 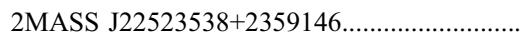 & $16.20 \pm 0.11$ & $15.91 \pm 0.20$ & 15.94(null) & 1997 Oct 6 & +28.6 \\
\hline 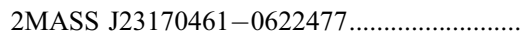 & $16.13 \pm 0.09$ & $15.96 \pm 0.19$ & 16.15(null) & 1998 Oct 10 & -1.6 \\
\hline 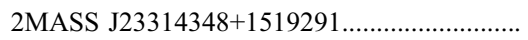 & $16.39 \pm 0.12$ & $16.22 \pm 0.20$ & 16.32(null) & 1997 Sep 29 & +16.8 \\
\hline 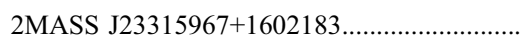 & $16.29 \pm 0.12$ & $16.26 \pm 0.21$ & 16.85(null) & 1997 Sep 29 & +17.5 \\
\hline 2MASS J23335590-0441137 ……..................... & $16.04 \pm 0.07$ & $15.77 \pm 0.12$ & $15.87 \pm 0.23$ & 2000 Sep 17 & -1.7 \\
\hline
\end{tabular}

a J2000.0 coordinates from the 2MASS All-Sky Point Source Catalog.

b Photometry from 2MASS All-Sky Point Source Catalog.

${ }^{c}$ UT date of 2MASS observation.

${ }^{\mathrm{d}}$ Ecliptic latitude.

followed-up $\sim 120$ of these objects, and we report on follow-up of three of these objects here: 2MASS 1106+27, 2MASS 1324+63, and 2MASS 1404-31 (see Table 3). 2MASS 1324+63 was independently identified as a T dwarf by S. Metchev et al. (2007, in preparation). Both 2MASS $1106+27$ and 2MASS 1404-31 are new discoveries. We also identified two previously discovered T dwarfs: 2MASS 0939-24 (T8; Tinney et al. 2005) and SDSSp 1346-00 (T6.5; Tsvetanov et al. 2000; see Table 4).

\subsection{Imaging: UH $2.2 \mathrm{~m}$ ULBCAM and IRTF $3.0 \mathrm{~m} \mathrm{SpeX}$}

We conducted follow-up imaging to cull the large number of transients from our 2MASS photometric search sample, using the UH $2.2 \mathrm{~m}$ ULBCAM and the IRTF $3.0 \mathrm{~m}^{6} \mathrm{SpeX}$ cameras. Our

\footnotetext{
${ }^{6}$ The $2.2 \mathrm{~m}$ and $3.0 \mathrm{~m}$ telescopes are both located on the summit of Mauna Kea, Hawai'i.
} 
TABLE 3

IRTF SPEX-PRISM LOG

\begin{tabular}{|c|c|c|c|c|c|c|c|c|c|}
\hline Object $^{\mathrm{a}}$ & $J^{\mathrm{b}}$ & $H^{\mathrm{b}}$ & $K_{s}^{\mathrm{b}}$ & UT Date & $\mathrm{AM}^{\mathrm{c}}$ & $\begin{array}{c}N \times t^{\mathrm{d}} \\
\text { (s) }\end{array}$ & $\begin{array}{c}\text { Spectral } \\
\text { Type }\end{array}$ & $\begin{array}{l}b / \beta^{\mathrm{e}} \\
(\mathrm{deg})\end{array}$ & Calibrator \\
\hline 2MASS J01072340+4759060... & $15.90 \pm 0.08$ & $16.11 \pm 0.18$ & $15.68 \pm 0.19$ & 2006 Aug 17 & 1.15 & $8 \times 120$ & M3 & $-14.8^{\circ} /+37.2^{\circ}$ & HD 9711 (A0 V) \\
\hline 2MASS J05063725-3405090....... & $16.36 \pm 0.13$ & $16.06 \pm 0.19$ & 16.22(null) & 2006 Dec 8 & 1.70 & $8 \times 120$ & M7 & $-35.5^{\circ} /-56.6^{\circ}$ & HD $29028(A 0 \mathrm{~V})$ \\
\hline 2MASS J05103520-4208140........... & $16.22 \pm 0.09$ & $16.24 \pm 0.16$ & $16.00 \pm 0.28$ & 2006 Sep 2 & 2.37 & $4 \times 120$ & $\mathrm{~T} 5$ & $-35.9^{\circ} /-64.6^{\circ}$ & HD $38921(\mathrm{~A} 0 \mathrm{~V})$ \\
\hline 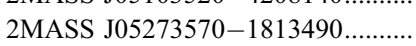 & $16.49 \pm 0.23$ & $16.53 \pm 0.39$ & $15.16($ null) & 2006 Sep 1 & 1.56 & $8 \times 120$ & M4 & $-26.4^{\circ} /-41.4^{\circ}$ & HD $37190(\mathrm{~A} 0 \mathrm{~V})$ \\
\hline 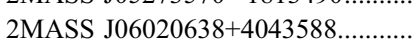 & $15.54 \pm 0.07$ & $15.59 \pm 0.14$ & $15.17 \pm 0.16$ & $2006 \operatorname{Dec} 9$ & 1.07 & $8 \times 120$ & $\mathrm{~T} 4.5$ & $+8.8^{\circ} /+17.3^{\circ}$ & HD $39250(\mathrm{~A} 0 \mathrm{~V})$ \\
\hline 2MASS J07290002-3954043 ........... & $15.92 \pm 0.08$ & $15.98 \pm 0.19$ & 15.29 (null) & 2006 Dec 8 & 2.00 & $16 \times 120$ & T8.0pec & $-10.4^{\circ} /-60.6^{\circ}$ & HD $61516(\mathrm{~A} 0 \mathrm{~V})$ \\
\hline SDSSp J083717.22-000018.3 .......... & $17.10 \pm 0.21$ & $15.99 \pm 0.17$ & 15.67 (null) & 2006 Dec 21 & 1.08 & $20 \times 180$ & T1 std & $+23.4^{\circ} /-17.9^{\circ}$ & HD $74721(\mathrm{~A} 0 \mathrm{~V})$ \\
\hline 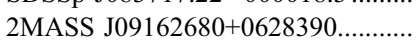 & $16.25 \pm 0.14$ & $16.29 \pm 0.26$ & 15.71 (null) & $2006 \operatorname{Dec} 9$ & 1.03 & $4 \times 120$ & M6.5 & $+35.1^{\circ} /-8.9^{\circ}$ & HD $79108(\mathrm{~A} 0 \mathrm{~V})$ \\
\hline 2MASS J09212890-0339410........... & $16.46 \pm 0.14$ & $16.53 \pm 0.30$ & 15.90 (null) & 2006 Dec 9 & 1.12 & $8 \times 120$ & M7: & $+30.8^{\circ} /-18.2^{\circ}$ & HD $79108(\mathrm{~A} 0 \mathrm{~V})$ \\
\hline 2MASS J09351549+0019103............ & $16.47 \pm 0.15$ & $16.27 \pm 0.20$ & 16.61(null) & 2006 Dec 21 & 1.11 & $4 \times 120$ & M7: & $+35.9^{\circ} /-13.3^{\circ}$ & HD $74721(\mathrm{~A} 0 \mathrm{~V})$ \\
\hline 2MASS J10073369-4555147........... & $15.65 \pm 0.07$ & $15.69 \pm 0.12$ & $15.56 \pm 0.23$ & 2006 Dec 9 & 2.45 & $8 \times 120$ & $\mathrm{~T} 5$ & $+8.0^{\circ} /-52.1^{\circ}$ & HD $88113(\mathrm{~A} 0 \mathrm{~V})$ \\
\hline 2MASS J11061197+2754225 ............ & $14.82 \pm 0.04$ & $14.15 \pm 0.05$ & $13.80 \pm 0.05$ & 2006 Apr 8 & 1.03 & $8 \times 120$ & $\mathrm{~T} 2.5$ & $+66.7^{\circ} /+20.3^{\circ}$ & HD $89239(\mathrm{~A} 0 \mathrm{~V})$ \\
\hline SDSS J120747.17+024424.8 $\ldots \ldots \ldots \ldots$ & $15.58 \pm 0.07$ & $14.56 \pm 0.07$ & $13.99 \pm 0.06$ & 2006 Dec 20 & 1.21 & $8 \times 120$ & T0 std & $+63.5^{\circ} /+3.3^{\circ}$ & HD $111744(\mathrm{~A} 0 \mathrm{~V})$ \\
\hline 2MASS J12154432-3420591_......... & $16.24 \pm 0.13$ & $15.81 \pm 0.19$ & 16.32(null) & 2006 May 31 & 2.05 & $8 \times 120$ & $\mathrm{~T} 4.5$ & $+27.9^{\circ} /-29.7^{\circ}$ & SAO $203194($ A0 V) \\
\hline 2MASS J13243559+6358284........... & $15.60 \pm 0.07$ & $14.58 \pm 0.06$ & $14.06 \pm 0.07$ & 2006 Apr 11 & 1.49 & $6 \times 150$ & $\mathrm{~T} 2.5$ & $+52.8^{\circ} /+62.6^{\circ}$ & HD $118214(\mathrm{~A} 0 \mathrm{~V})$ \\
\hline 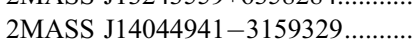 & $15.58 \pm 0.07$ & $14.96 \pm 0.08$ & $14.54 \pm 0.10$ & 2006 Jun 1 & 2.18 & $12 \times 120$ & $\mathrm{~T} 2.5$ & $+28.4^{\circ} /-18.1^{\circ}$ & SAO $205525(\mathrm{~A} 0 \mathrm{~V})$ \\
\hline 2MASS J16150413+1340079....... & $16.35 \pm 0.09$ & $16.49 \pm 0.25$ & 15.86(null) & 2006 Sep 2 & 1.10 & $16 \times 120$ & T6 & $+48.8^{\circ} /+34.3^{\circ}$ & $\mathrm{q} \operatorname{Her}(\mathrm{A} 0 \mathrm{~V})$ \\
\hline 2MASS J16293700+1415440.......... & $16.27 \pm 0.11$ & $16.36 \pm 0.26$ & $15.00 \pm 0.15$ & 2006 Sep 2 & 1.32 & $4 \times 120$ & M6: & $+37.8^{\circ} /+35.6^{\circ}$ & q Her (A0 V) \\
\hline 2MASS J20014670-3805400............ & $16.42 \pm 0.11$ & $16.44 \pm 0.23$ & 15.87 (null) & 2006 Sep 1 & 2.10 & $8 \times 120$ & M4 & $-29.5^{\circ} /-17.2^{\circ}$ & HD $189501(\mathrm{~A} 0 \mathrm{~V})$ \\
\hline 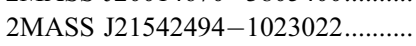 & $16.43 \pm 0.12$ & $16.45 \pm 0.28$ & 17.05 (null) & 2006 Aug 17 & 1.28 & $8 \times 120$ & $\mathrm{~T} 4.5$ & $-45.2^{\circ} /+2.2^{\circ}$ & HD $211278(\mathrm{~A} 0 \mathrm{~V})$ \\
\hline 2MASS J21543318+5942187............ & $15.66 \pm 0.07$ & $15.77 \pm 0.17$ & 15.34(null) & 2006 Nov 17 & 1.41 & $12 \times 180$ & $\mathrm{~T} 5$ & $+4.1^{\circ} /+63.7^{\circ}$ & $\mathrm{BD}+622148(\mathrm{~A} 0 \mathrm{~V})$ \\
\hline 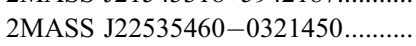 & $16.01 \pm 0.09$ & $16.09 \pm 0.22$ & $15.46 \pm 0.23$ & 2006 Aug 18 & 1.16 & $8 \times 120$ & M6 & $-53.2^{\circ} /+3.4^{\circ}$ & HD $216807(\mathrm{~A} 0 \mathrm{~V})$ \\
\hline
\end{tabular}

\footnotetext{
a J2000.0 coordinates from the 2MASS All-Sky Point Source Catalog.

b Photometry from 2MASS All-Sky Point Source Catalog.

c Air mass.

${ }^{d}$ Number of integrations times integration time.

${ }^{\mathrm{e}}(b)$ Galactic latitude; $(\beta)$ ecliptic latitude.
}

TABLE 4

Spectrophotometric Properties of New and Confirmed T Dwarfs

\begin{tabular}{|c|c|c|c|c|c|c|c|c|}
\hline Object & $J^{\mathrm{a}}$ & $J-H^{\mathrm{a}}$ & $H-K_{s}{ }^{\mathrm{a}}$ & $J-K_{s}^{\mathrm{a}}$ & $i^{\mathrm{b}}$ & $z^{\mathrm{b}}$ & Spectral Type & $\begin{array}{l}\text { Distance }^{\mathrm{c}} \\
\quad(\mathrm{pc})\end{array}$ \\
\hline \multicolumn{9}{|c|}{ New T Dwarfs } \\
\hline 2MASS J05103520-4208140............. & $16.22 \pm 0.09$ & $-0.02 \pm 0.18$ & $0.24 \pm 0.32$ & $0.23 \pm 0.30$ & $\cdots$ & $\cdots$ & $\mathrm{T} 5$ & $24.3 \pm 2.0$ \\
\hline 2MASS J06020638+4043588.............. & $15.54 \pm 0.07$ & $-0.05 \pm 0.15$ & $0.43 \pm 0.21$ & $0.38 \pm 0.17$ & $\ldots$ & $\ldots$ & $\mathrm{T} 4.5$ & $19.3 \pm 1.5$ \\
\hline 2MASS J07290002-3954043............. & $15.92 \pm 0.08$ & $-0.06 \pm 0.20$ & $<0.69$ & $<0.63$ & $\ldots$ & $\ldots$ & T8pec & $8.4 \pm 0.7$ \\
\hline 2MASS J10073369-4555147............. & $15.65 \pm 0.07$ & $-0.04 \pm 0.14$ & $0.13 \pm 0.26$ & $0.09 \pm 0.24$ & $\ldots$ & $\ldots$ & T5 & $18.7 \pm 1.4$ \\
\hline 2MASS J11061197+2754225 .............. & $14.82 \pm 0.04$ & $0.67 \pm 0.06$ & $0.35 \pm 0.07$ & $1.02 \pm 0.06$ & $21.28 \pm 0.09$ & $17.70 \pm 0.03$ & $\mathrm{~T} 2.5$ & $15.5 \pm 1.2$ \\
\hline 2MASS J12154432-3420591_............ & $16.24 \pm 0.13$ & $0.43 \pm 0.23$ & $<-0.51$ & $<-0.08$ & $\ldots$ & & $\mathrm{T} 4.5$ & $26.6 \pm 2.1$ \\
\hline 2MASS J13243559+6358284_............. & $15.60 \pm 0.07$ & $1.02 \pm 0.10$ & $0.52 \pm 0.09$ & $1.54 \pm 0.10$ & $22.68 \pm 0.26$ & $18.73 \pm 0.04$ & T2:pec & $21.8 \pm 1.7$ \\
\hline 2MASS J14044941-3159329............ & $15.58 \pm 0.07$ & $0.62 \pm 0.10$ & $0.42 \pm 0.12$ & $1.04 \pm 0.12$ & $\ldots$ & & $\mathrm{T} 2.5$ & $22.0 \pm 1.7$ \\
\hline 2MASS J16150413+1340079............. & $16.35 \pm 0.09$ & $-0.14 \pm 0.27$ & $<0.63$ & $<0.49$ & $\ldots$ & $19.90 \pm 0.09$ & T6 & $20.4 \pm 1.6$ \\
\hline 2MASS J21542494-1023022 …......... & $16.43 \pm 0.12$ & $-0.03 \pm 0.30$ & $<-0.59$ & $<-0.62$ & $\ldots$ & $\ldots$ & $\mathrm{T} 4.5$ & $29.0 \pm 2.3$ \\
\hline 2MASS J21543318+5942187................ & $15.66 \pm 0.07$ & $-0.10 \pm 0.18$ & $<0.43$ & $<0.32$ & $\ldots$ & $\ldots$ & T6 & $18.8 \pm 1.5$ \\
\hline \multicolumn{9}{|c|}{ Recovered T Dwarfs } \\
\hline 2MASS J09393548-2448279 ${ }^{\mathrm{d}}$. & $15.98 \pm 0.11$ & $0.18 \pm 0.18$ & $<-0.76$ & $<-0.58$ & $\ldots$ & $\cdots$ & $\mathrm{T} 8^{\mathrm{e}}$ & $8.7 \pm 0.7$ \\
\hline SDSSp J134646.45-003150.4 $\ldots$ & $16.00 \pm 0.10$ & $0.54 \pm 0.16$ & $-0.31 \pm 0.30$ & $0.23 \pm 0.29$ & $\ldots$ & $\ldots$ & $\mathrm{T} 6.5^{\mathrm{e}}$ & $15.0 \pm 1.2$ \\
\hline SDSS J163022.92+081822. $0^{\mathrm{d}} \ldots \ldots$ & $16.40 \pm 0.11$ & $0.07 \pm 0.31$ & $<-0.28$ & $<-0.21$ & $23.82 \pm 0.61$ & $20.13 \pm 0.10$ & $\mathrm{~T} 5.5^{\mathrm{e}}$ & $23.7 \pm 1.8$ \\
\hline SDSS J175805.46+463311. $9^{\mathrm{d}}$ & $16.15 \pm 0.09$ & $-0.10 \pm 0.24$ & $0.79 \pm 0.29$ & $0.69 \pm 0.21$ & $24.18 \pm 0.57$ & $19.67 \pm 0.07$ & $\mathrm{~T} 6.5^{\mathrm{e}}$ & $16.1 \pm 1.2$ \\
\hline SDSS J212413.89+010000.3 ${ }^{\mathrm{d}}$ & $16.03 \pm 0.07$ & $-0.15 \pm 0.21$ & $<0.04$ & $<-0.11$ & $23.77 \pm 0.54$ & $19.71 \pm 0.12$ & $\mathrm{~T}^{\mathrm{e}}$ & $22.3 \pm 1.7$ \\
\hline
\end{tabular}

${ }^{\text {a }}$ Photometry from 2MASS All-Sky Point Source Catalog.

${ }^{\mathrm{b}}$ Photometry from SDSS (AB) Data Release 5.

c Spectrophotometric distance estimates derived in $\S 3.2 .1$.

d References: 2MASS 0939-24 (Tinney et al. 2005), SDSS 1346-00 (Tsvetanov et al. 2000), SDSS 1630+08 (Chiu et al. 2006), SDSS 1758+46 (Knapp et al. 2004), SDSS 2124+01 (Knapp et al. 2004).

e Burgasser et al. (2006b). 


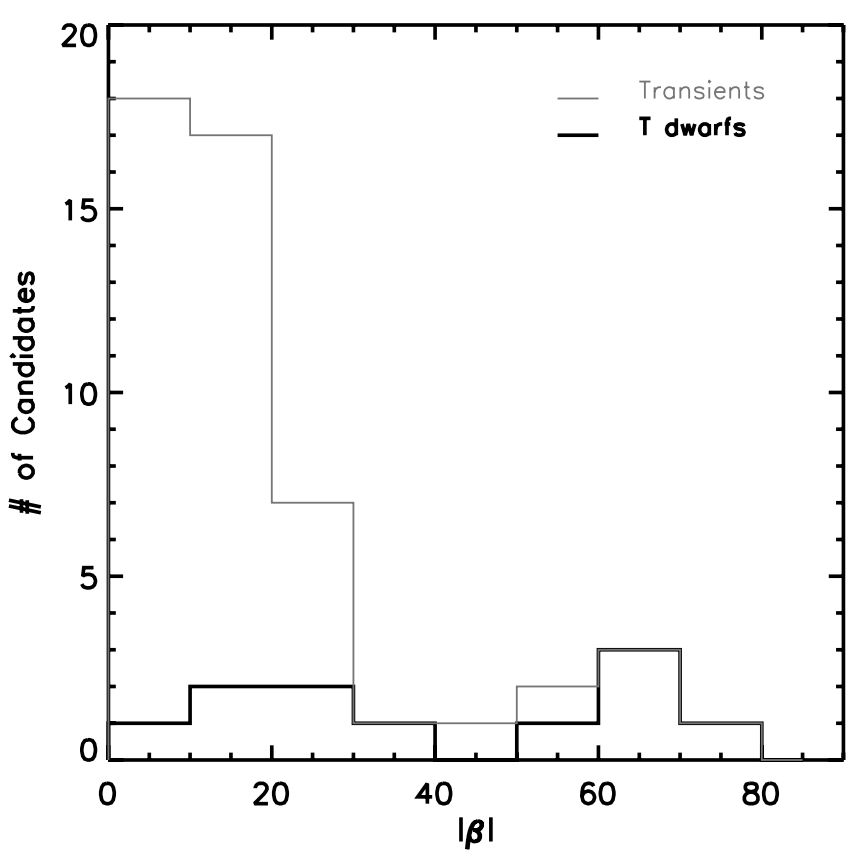

Fig. 2.- Histogram for all 50 transients (gray, light line) and all eight new $\mathrm{T}$ dwarfs and three recovered T dwarfs (black, heavy line) identified in the 2MASS photometric search for mid- to late-type T dwarfs, as a function of ecliptic latitude, $|\beta|$. Note that while the $\mathrm{T}$ dwarfs are distributed nearly evenly across ecliptic latitutudes, the vast majority of transients are concentrated near the ecliptic, at $|\beta| \leq 30^{\circ}$.

2MASS proper-motion search sample did not require follow-up imaging, since second epoch 2MASS images confirmed the existence of our targets. We imaged a total of 67 candidates during several runs described below.

The Ultra Low Background Camera (ULBCAM) ${ }^{7}$ is a mosaic infrared camera with four $2048 \times 2048$ arrays, a $17^{\prime} \times 17^{\prime}$ field of view (FOV), and a $0.25^{\prime \prime}$ pixel scale. However, we used only the top right array, with an individual FOV of $8^{\prime} 32^{\prime \prime} \times 8^{\prime} 32^{\prime \prime}$, because it has the highest quantum efficiency and lowest number of bad pixels of the four arrays. We imaged candidates with ULBCAM on 2006 February 9-12 and 16 UT and July 24 UT with clear and photometric conditions on all nights and good seeing ( $\lesssim 1^{\prime \prime}$ at the $J$ band). We used the $J$-band filter for all observations and dithered between exposures, where individual exposure times varied from 20 to $180 \mathrm{~s}$. All images were first bias-subtracted and then pair-wise subtracted to give the final image. All resultant images were deeper than the 2MASS $J$-band image for the same field.

SpeX has a $512 \times 512$ array InSb camera with a $60^{\prime \prime} \times 60^{\prime \prime}$ FOV and a $0.12^{\prime \prime}$ pixel scale (Rayner et al. 2003). We used this camera to image candidates during 2006 May 31 UT, August 1718 UT, September 1-2 UT, November 17 UT, and December 8 9 and 20-21 UT. Conditions for the 2006 May 31 UT run were clear, with $1^{\prime \prime}$ seeing at $J$ band. Conditions were also clear, with $0.6^{\prime \prime}-0.7^{\prime \prime}$ seeing at $J$ band, for the 2006 August $17-18$ UT and September 1-2 UT runs. Conditions for the 2006 November 17 run ranged from clear to light cirrus with $1.0^{\prime \prime}$ seeing at $K$ band and similar conditions for the 2006 December 8-9 and 20-21 UT runs with $0.5^{\prime \prime}-0.8^{\prime \prime}$ seeing at $K$ band. Using the $J$-band filter, we took $60 \mathrm{~s}$ exposures with a single offset for pair-wise subtraction. In all cases where neighboring stars fell in the camera's FOV, we were able to verify that the resulting images were deeper than the 2MASS $J$-band image for the same field. Candidates absent in our two imaging campaigns are listed in Table 2.

\subsection{Spectroscopy: IRTF $3.0 \mathrm{~m} \mathrm{SpeX}$}

Spectroscopic observations were obtained using the IRTF SpeX spectrograph in low-resolution mode, covering $\sim 0.7-2.5 \mu \mathrm{m}$ in a single order on the $1024 \times 1024 \mathrm{InSb}$ array. We used the $0.5^{\prime \prime}$ slit, resulting in $R \sim 150$, and rotated the slit to the parallactic angle to minimize slit losses. For accurate sky subtraction, we nodded along the slit in ABBA cycles. Exposure times for science targets ranged from 120 to $180 \mathrm{~s}$ to maximize the signal-tonoise ratio and to minimize temporal variations of $\mathrm{OH}$ airglow, which can leave a forest of residual lines for long exposures (see Figs. 1, 2, and 3 of Ramsay et al. 1992).

We obtained spectroscopic observations for a total of 20 science targets, $2 \mathrm{~T}$ dwarf standards, and 20 calibrator A0 V stars between 2006 April 8 UT and December 21 UT. We list a log of our observations in Table 3. All nights were clear or had light cirrus. All calibrator A0 stars were observed either immediately before or after the science target with a differential air mass less than 0.10 for accurate flux calibration. After observing the calibrator stars, we immediately took internal flat-field and argon arc lamps for instrumental calibration. We employed standard reduction techniques using the Spextool package version 3.2 (Cushing et al. 2004; Vacca et al. 2003).

\section{ANALYSIS \\ 3.1. $M$ Dwarfs}

NIR spectroscopy of candidates confirmed via second-epoch imaging (see $\S 2.2$ ) reveals nine of them as M dwarfs. To classify these spectra, we compared their overall morphology, particularly the strength of their $\mathrm{H}_{2} \mathrm{O}$ absorptions at $1.30-1.51$ and $1.75-2.05 \mu \mathrm{m}$, to a set of known M dwarfs: Gl 229A (M1), Gl 411 (M2), Gl 388 (M3), Gl 213 (M4), Gl 51 (M5), Gl 406 (M6), vB8 (M7), vB10 (M8), LHS 2924 (M9), and BRI 0021-0214 (M9.5) (Cushing et al. 2005). We list the spectral types (M3M7) of these nine M dwarfs in Table 3 and estimate our accuracy as \pm 1 subtype, except for those types denoted with a colon due to spectra with a poor signal-to-noise ratio. None of these nine $\mathrm{M}$ dwarf spectra differ markedly from our comparison $\mathrm{M}$ dwarfs, suggesting that the unusual NIR colors of these M dwarfs shown in Figure 1 are due to large photometric errors instead of spectroscopic peculiarities.

\section{2. $T$ Dwarfs}

\subsubsection{Classification and Kinematics}

In Figure 3 we show finder charts for all 11 new T dwarfs constructed from 2MASS All-Sky Release Survey ${ }^{8} J$-band images. We classified these $T$ dwarfs by comparing their overall spectral morphologies to NIR T dwarf primary standards (see Fig. 4) defined by Burgasser et al. (2006b): SDSS J120747.17+024424.8 (T0 std), SDSSp J083717.22-000018.3 (T1 std), SDSSp J125453.90-012247.4 (T2 std), 2MASS J12095613-1004008 (T3 std), 2MASSI J2254188+312349 (T4 std), 2MASS J15031961+2525196 (T5 std), SDSSp J162414.37+002915.6 (T6 std), 2MASSI J0727182+171001 (T7 std), and 2MASSI J0415195-093506 (T8 std). These were also observed with IRTF SpeX using the $0.5^{\prime \prime}$ slit, so they have identical resolution ( $R \sim 150$ ) to our spectra (Burgasser et al. 2006b; this paper). We calculated NIR $\mathrm{H}_{2} \mathrm{O}$ and $\mathrm{CH}_{4}$ spectral indices defined in Burgasser et al. (2006b) for the 11 new T dwarfs (see Table 5). The indirect spectral types (calculated by comparing each index measurement to that of each standard and finding the nearest

\footnotetext{
${ }^{7}$ See http://www.ifa.hawaii.edu/88inch/2.2-meter-technical.htm.
}

\footnotetext{
${ }^{8}$ See http://irsa.ipac.caltech.edu/Missions/2mass.html.
} 

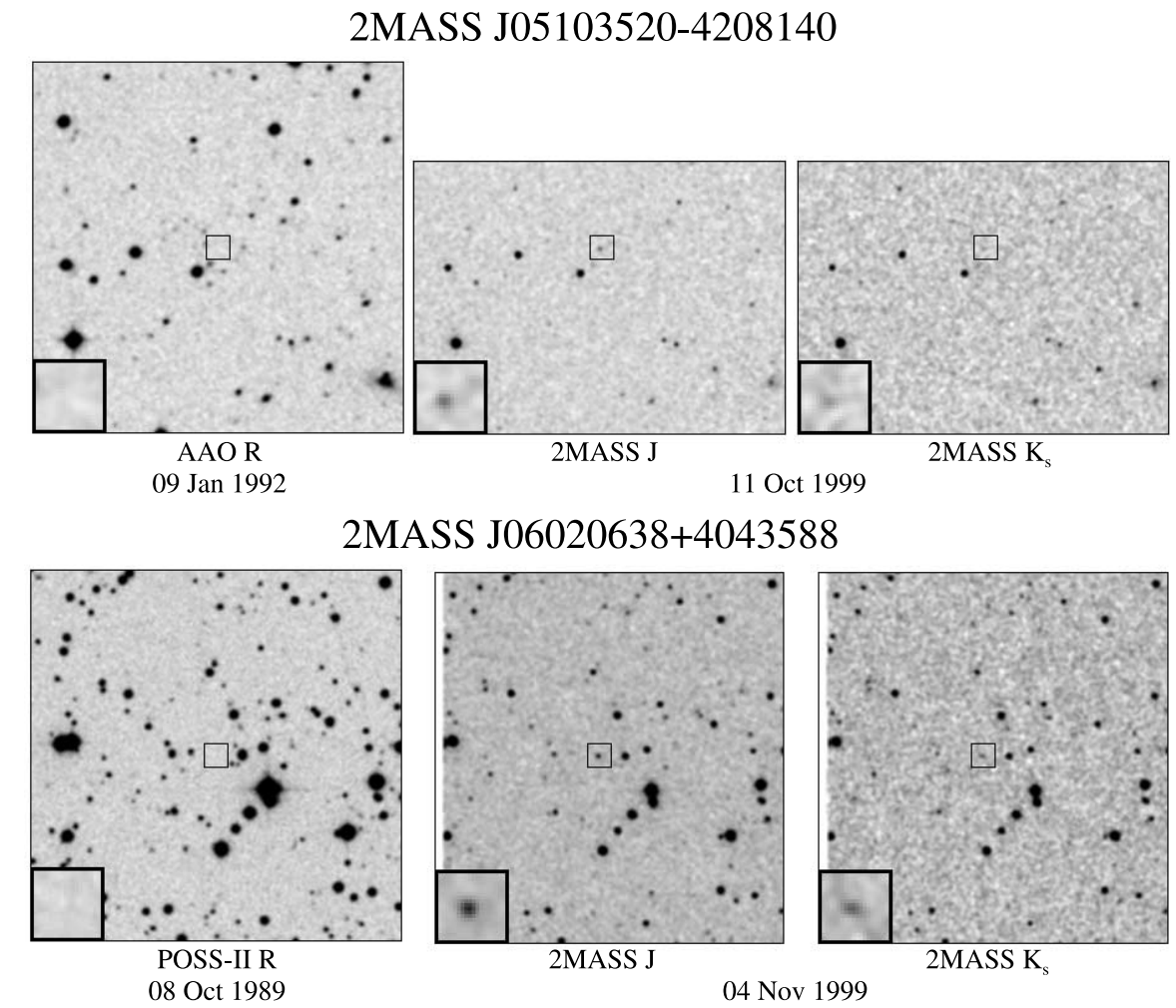

2MASS J07290002-3954043

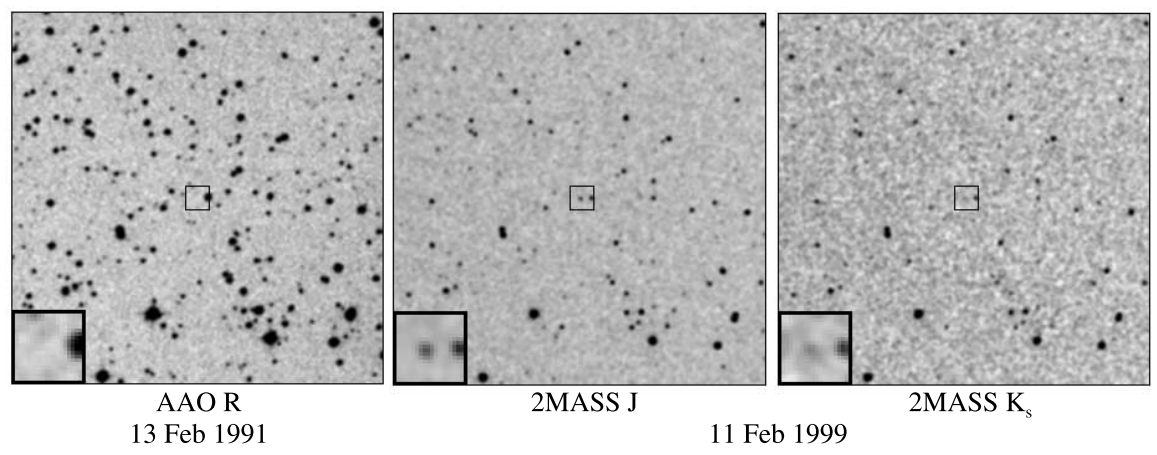

2MASS J10073369-4555147

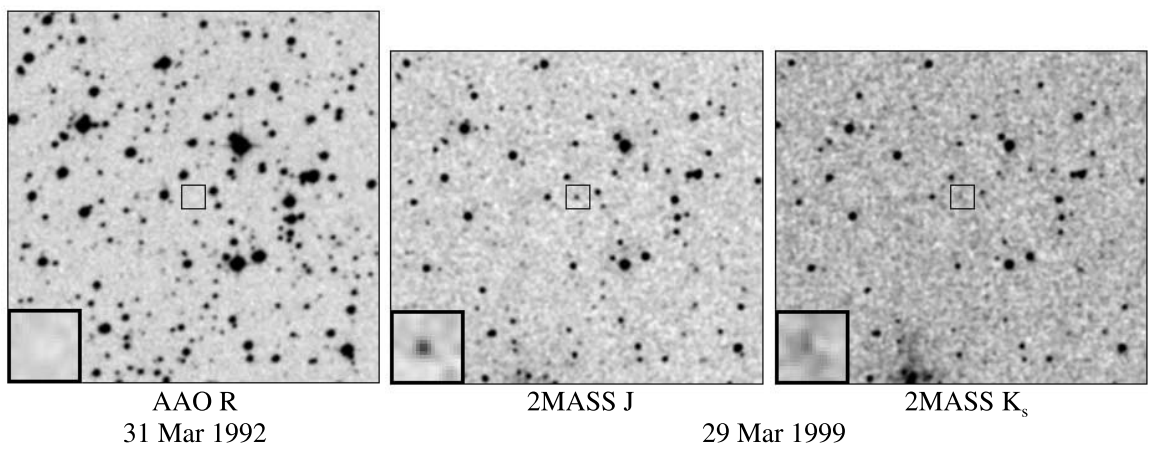

FIG. 3.-Finder charts for the 11 new T dwarfs constructed from DSS II $R$-band, 2MASS $J$-band, and 2 MASS $K_{s}$-band images. Each image is nominally $300^{\prime \prime}$ on a

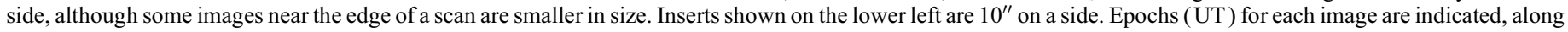
with the $\mathrm{J} 2000.0$ coordinates for each object represented by hhmm $\pm \mathrm{ddmm}$. 

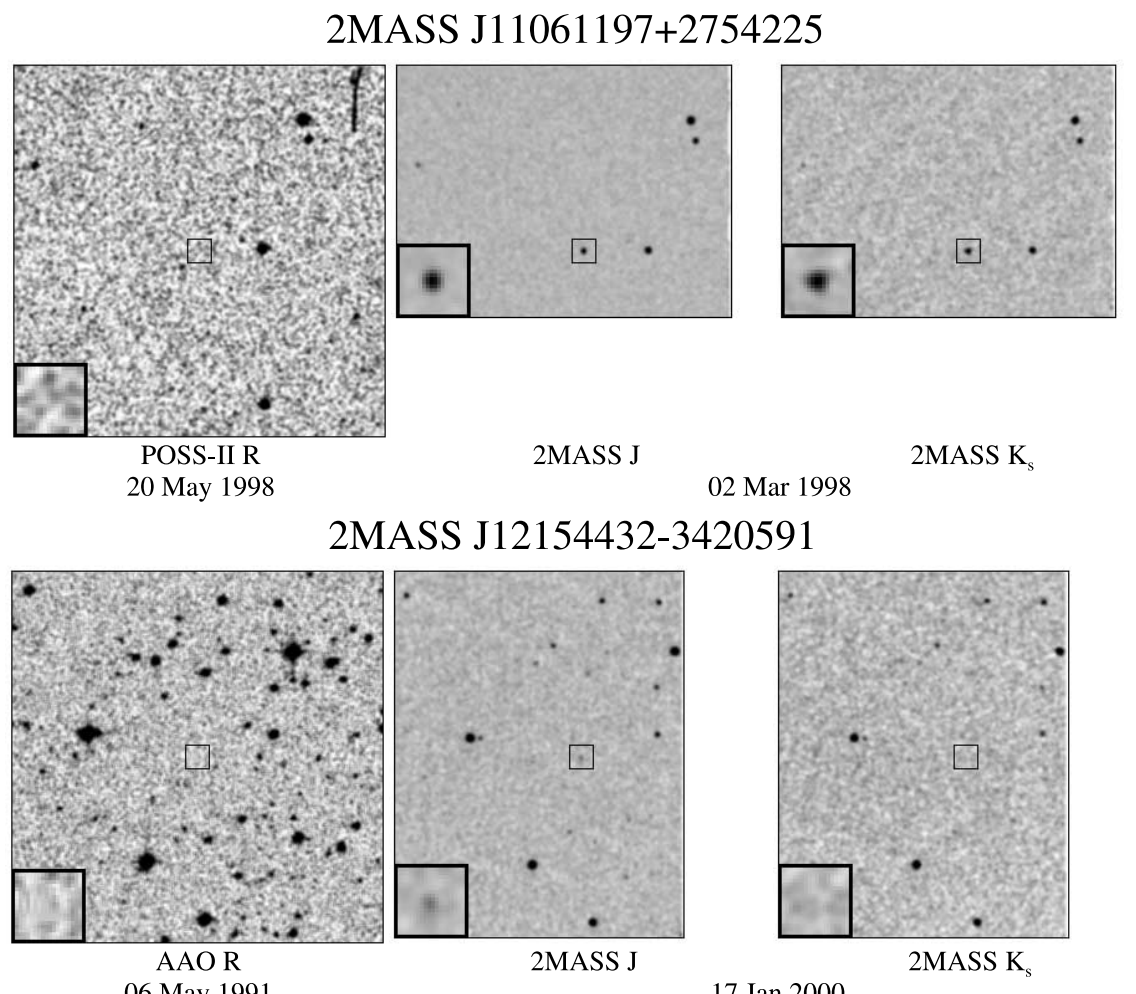

2MASS J13243559+6358284

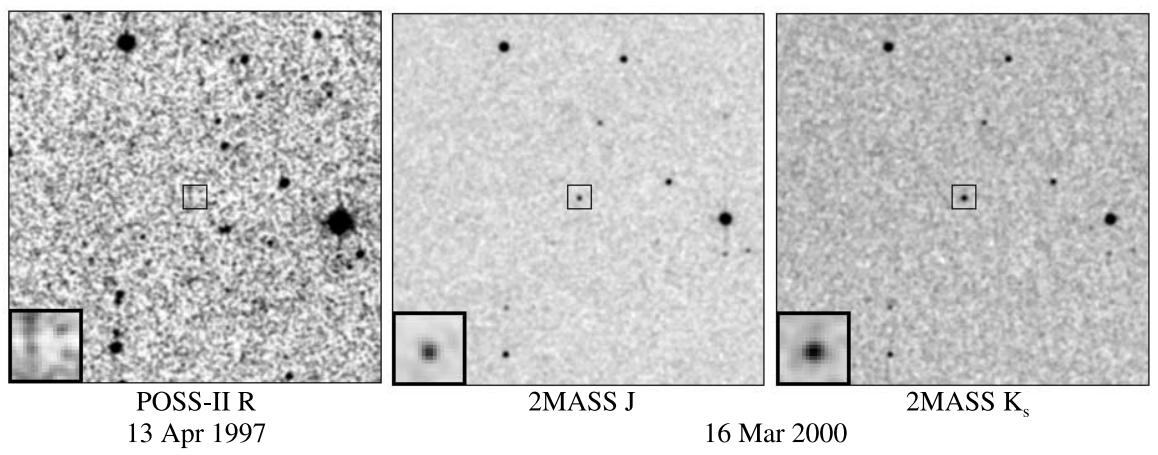

2MASS J14044941-3159329

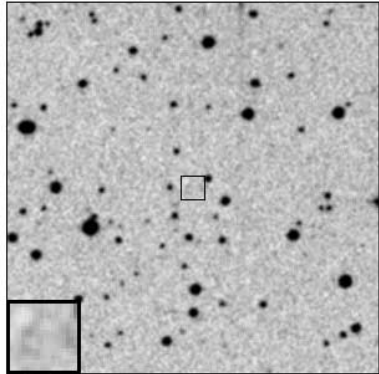

AAO R 13 Mar 1992

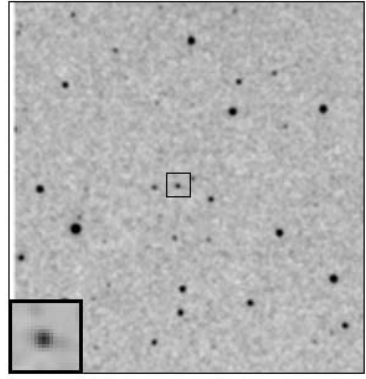

2MASS J

17 Jul 1998

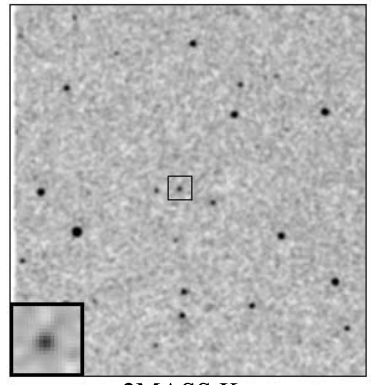

2MASS K

FIg. 3-Continued 


\section{MASS J16150413+1340079}
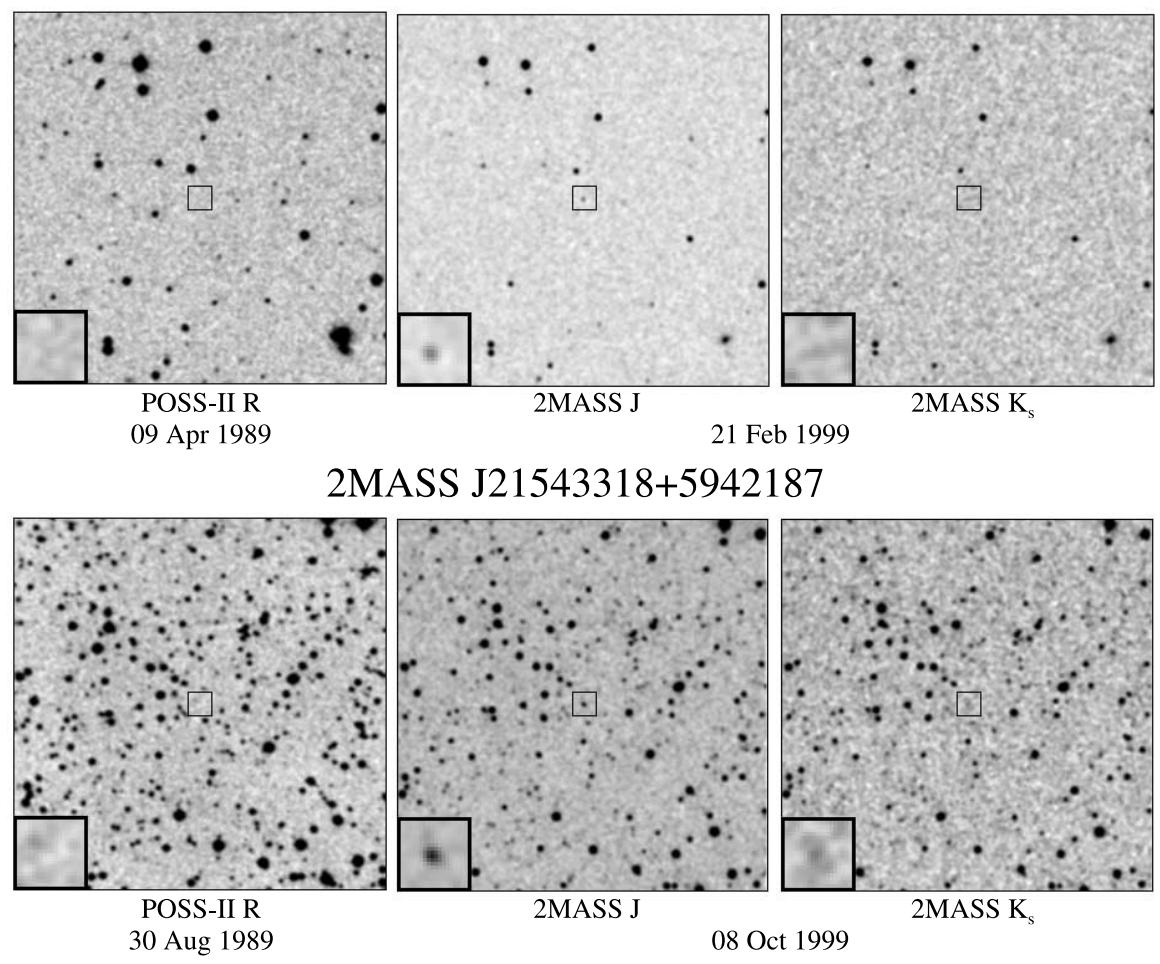

2MASS J21542494-1023022

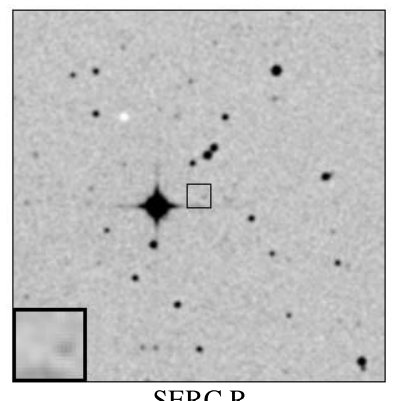

14 Oct 1987
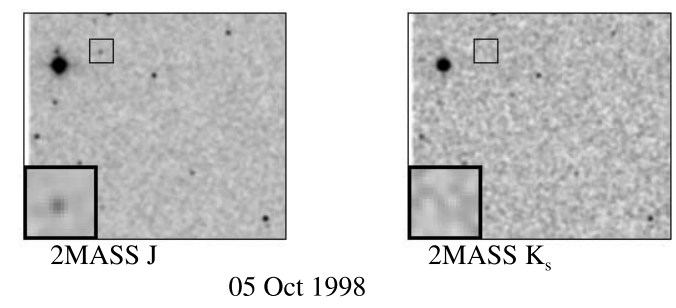

FIg. 3-Continued 

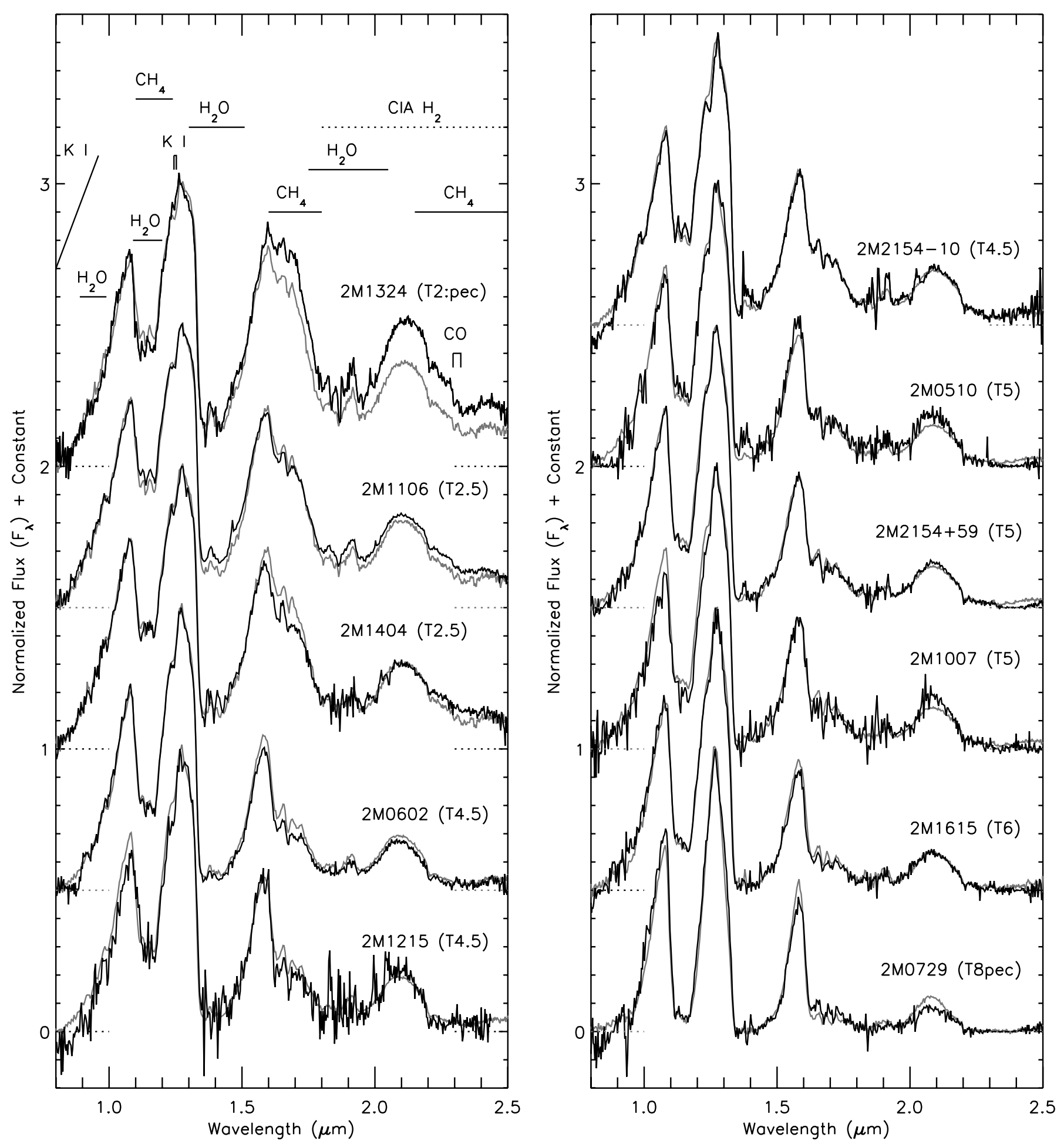

FIG. 4.- IRTF SpeX prism $(R \sim 150)$ spectra of the 11 newly discovered T dwarfs (thick black lines) overplotted on their nearest NIR spectral type match (thin gray lines). Integer spectral types are the NIR primary T dwarf standards defined by Burgasser et al. (2006b). Half-subtypes are from the synthetic spectral library (see $\S 3.2$ ). All spectra have been normalized at $1.27 \mu \mathrm{m}$ using a $0.01 \mu \mathrm{m}$ window and are offset by half-integer values (dotted lines show zero levels) for clarity. We have indicated the key atomic and molecular features. 
TABLE 5

Spectral Indices of 11 New T Dwarfs

\begin{tabular}{|c|c|c|c|c|c|c|c|c|c|}
\hline \multirow[b]{2}{*}{ Овјест } & \multirow[b]{2}{*}{$\mathrm{H}_{2} \mathrm{O}-J$} & \multirow[b]{2}{*}{$\mathrm{CH}_{4}-J$} & \multirow[b]{2}{*}{$\mathrm{H}_{2} \mathrm{O}-\mathrm{H}$} & \multirow[b]{2}{*}{$\mathrm{CH}_{4}-\mathrm{H}$} & \multirow[b]{2}{*}{$\mathrm{CH}_{4}-\mathrm{K}$} & \multirow[b]{2}{*}{$\mathrm{H} 2 \mathrm{O}-\mathrm{K}$} & \multirow[b]{2}{*}{$K / J$} & \multicolumn{2}{|c|}{ Derived NIR Spectral Type } \\
\hline & & & & & & & & Direct $^{\mathrm{a}, \mathrm{b}}$ & Ind $1^{\mathrm{b}, \mathrm{c}, \mathrm{d}}$ \\
\hline 2MASS J1324+63..................... & $0.431(\mathrm{~T} 2.5)$ & $0.529(\mathrm{~T} 3)$ & $0.485(\mathrm{~T} 2)$ & 1.006 (L9.5) & $0.665(\mathrm{~T} 1.5)$ & 0.589 & 0.501 & $\mathrm{~T} 2: \mathrm{pec}$ & T1.5: \\
\hline 2MASS J1106+27...................... & $0.481(\mathrm{~T} 2)$ & $0.564(\mathrm{~T} 2.5)$ & $0.522(\mathrm{~T} 1.5)$ & $0.781(\mathrm{~T} 2.5)$ & $0.614(\mathrm{~T} 2)$ & 0.585 & 0.336 & $\mathrm{~T} 2.5$ & $\mathrm{~T} 2$ \\
\hline 2MASS J1404-31 ................... & $0.442(\mathrm{~T} 2.5)$ & $0.522(\mathrm{~T} 3)$ & $0.506(\mathrm{~T} 1.5)$ & $0.749(\mathrm{~T} 3)$ & $0.649(\mathrm{~T} 1.5)$ & 0.613 & 0.312 & $\mathrm{~T} 2.5$ & T2.5 \\
\hline 2MASS J2154-10 ……............. & $0.349(\mathrm{~T} 4)$ & $0.438(\mathrm{~T} 4.5)$ & $0.395(\mathrm{~T} 4)$ & $0.524(\mathrm{~T} 4.5)$ & $0.273(\mathrm{~T} 4.5)$ & 0.498 & 0.212 & $\mathrm{~T} 4.5$ & T4.5 \\
\hline 2MASS J0602+40.................... & $0.303(\mathrm{~T} 4.5)$ & $0.433(\mathrm{~T} 4.5)$ & $0.363(\mathrm{~T} 4.5)$ & $0.457(\mathrm{~T} 4.5)$ & $0.240(\mathrm{~T} 4.5)$ & 0.469 & 0.181 & $\mathrm{~T} 4.5$ & $\mathrm{~T} 4.5$ \\
\hline 2MASS J1215-34.................... & $0.308(\mathrm{~T} 4.5)$ & $0.486(\mathrm{~T} 4)$ & $0.382(\mathrm{~T} 4)$ & $0.414(\mathrm{~T} 5)$ & $0.151(\mathrm{~T} 6)$ & 0.536 & 0.225 & $\mathrm{~T} 4.5$ & $\mathrm{~T} 4.5$ \\
\hline 2MASS J0510-42 …................ & $0.261(\mathrm{~T} 5)$ & $0.414(\mathrm{~T} 4.5)$ & $0.337(\mathrm{~T} 5)$ & $0.348(\mathrm{~T} 5.5)$ & $0.125(\mathrm{~T} 6.5)$ & 0.459 & 0.189 & T5 & T5.5 \\
\hline 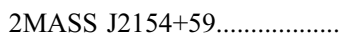 & $0.217(\mathrm{~T} 5.5)$ & $0.354(\mathrm{~T} 6)$ & $0.325(\mathrm{~T} 5.5)$ & $0.361(\mathrm{~T} 5.5)$ & $0.163(\mathrm{~T} 5.5)$ & 0.437 & 0.167 & $\mathrm{~T} 5$ & T5.5 \\
\hline 2MASS J1007-45 ..................... & $0.205(\mathrm{~T} 5.5)$ & $0.360(\mathrm{~T} 5)$ & $0.310(\mathrm{~T} 5.5)$ & $0.314(\mathrm{~T} 6)$ & $0.151(\mathrm{~T} 6)$ & 0.402 & 0.199 & T5 & T5.5 \\
\hline 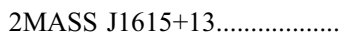 & $0.167(\mathrm{~T} 6)$ & $0.313(\mathrm{~T} 6.5)$ & $0.305(\mathrm{~T} 5.5)$ & $0.267(\mathrm{~T} 6.5)$ & $0.153(\mathrm{~T} 6)$ & 0.399 & 0.145 & $\mathrm{~T} 6$ & T6 \\
\hline 2MASS J0729-39 & $0.053(\mathrm{~T} 7.5)$ & $0.251(\mathrm{~T} 7)$ & $0.175(>\mathrm{T} 8)$ & $0.202(\mathrm{~T} 7)$ & $0.044(>\mathrm{T} 8)$ & 0.325 & 0.091 & T8pec & $\mathrm{T} 7$ \\
\hline
\end{tabular}

NotE.-NIR indices are defined by Burgasser et al. (2006b).

a Direct spectral typing is done by visually comparing objects against standards.

b Colons mark estimates with standard deviations $\geq 1$ spectral type.

c Index values outside the proscribed range (i.e., less than or greater than) are not used to compute the average spectral type.

d Spectral typing follows the convention of Burgasser et al. (2006b), where Ind 1 (in parentheses) is computed by comparison of the indices to the indices of standards.

subtype or half-subtype match) are in excellent agreement $(\leq 0.5$ subtypes) with our direct spectral classification except for 2MASS 0729-39, which has two indices that are classified as $>\mathrm{T} 8$ and are not included in the mean indirect spectral type.

We find a spectral type range of T2 to T8 and a wide range in color of $-0.6 \lesssim J-K_{s} \lesssim 1.5$. We were able to identify earlytype T dwarfs in 2MASS because we did not rely on NIR color selections in our proper-motion search. With the exception of 2MASS 1324+63 (T2:pec) and 2MASS 0729-39 (T8pec), these T dwarfs show $\mathrm{CH}_{4}$ and $\mathrm{H}_{2} \mathrm{O}$ absorption strengths throughout the NIR regime consistent with standards of similar spectral types (see Fig. 4). In Figure 5 we show the NIR $\left(J, H, K_{s}\right)$ colors along with optical $(i, z)$ colors, when available, of these $11 \mathrm{~T}$ dwarfs in comparison to all known $\mathrm{T}$ dwarfs with individual magnitude errors of less than 0.3 mag.

The NIR colors of these new T dwarfs span a broad range, but generally track with the trend in color as a function of spectral type. While 2MASS 1324+63 lies along the redder edge of the dispersal relative to objects of similar spectral type and 2MASS 0729-39 lies along the bluer edge relative to objects of similar spectral type, they are both within $1 \sigma$ of their own photometric errors from other sources in the dispersion.

Although the bottom panel of far optical minus NIR colors has very few sources, 2MASS $1324+63$ is indistinct from the scatter of other T2 dwarfs. On the other hand, 2MASS $1106+27$ is the only T2.5 dwarf with a measured $i$-band color in SDSS but is distinctly $(>1 \sigma)$ bluer than all T2 dwarfs and is the bluest T2.5 dwarf in $z-J$. Although 2MASS $1106+27$ is the second brightest $\mathrm{T}$ dwarf in SDSS $(z=17.70 \pm 0.03$; see Table 4), it was not found in previous searches of SDSS for T dwarfs because the photometry for a nearby faint source was registered instead. To get the SDSS colors for this object, we had to use the Navigate Visual Tool ${ }^{9}$ to find 2MASS 1106+27. We used the Explore radio button and recorded the parameters of this object, which we fed to an SQL query, requesting the point-spread function magnitudes (see Table 4). We obtained the $i$ and $z$ magnitudes of 2MASS 1324+63 and 2MASS 1615+13 from the SDSS Catalog in a standard fashion, requesting the "psf_mags" and "psf_magerrs" (see Table 4).

\footnotetext{
${ }^{9}$ See http://cas.sdss.org/astrodr5/en/tools/chart/navi.asp.
}

Spectrophotometric distance estimates were calculated using the spectral types derived above and the Liu et al. (2006) spectral type versus magnitude relation (excluding known binaries) and are given along with other spectrophotometric properties in Table 4 . These distances range from $\sim 8.4$ to $29.0 \mathrm{pc}$. For the three earlytype T dwarfs from the 2MASS proper-motion survey, we were able to calculate proper motions and position angles from the multiepoch data (see Table 6 for kinematic properties). We were also able to do the same for 2MASS $1615+13$, which was detected in the $z$ band in the SDSS Catalog. The proper motions along with the distance estimates yielded tangential velocity estimates of $\sim 36-66 \mathrm{~km} \mathrm{~s}^{-1}$ for these four objects, which are near or exceeding the median $V_{\tan }=39.0 \mathrm{~km} \mathrm{~s}^{-1}$ for T dwarfs found by Vrba et al. (2004). The tangential velocities of these four $\mathrm{T}$ dwarfs are nearly twice that of the median tangential velocities of L dwarfs ( $V_{\tan }=24.5 \mathrm{~km} \mathrm{~s}^{-1}$; Vrba et al. 2004). Our proper-motion sample is biased toward these higher velocity objects; i.e., at a distance of $20 \mathrm{pc}$ we would only detect objects with $V_{\tan } \gtrsim 4.74 \times 0.4^{\prime \prime} \mathrm{yr}^{-1} \times 20 \mathrm{pc}=38 \mathrm{~km} \mathrm{~s}^{-1}$. We use $0.4^{\prime \prime} \mathrm{yr}^{-1}$ in this estimate, since $0.4^{\prime \prime}$ is the motion floor for the survey and the mean epoch difference is near $1 \mathrm{yr}$.

\subsection{Spectroscopically Peculiar Sources}

Two of the $\mathrm{T}$ dwarfs identified in this study have spectral properties that are somewhat unusual compared to the spectral standards. We discuss these peculiar sources in detail.

\subsubsection{MASS 1324+63 (T2:pec)}

While the spectrum of 2MASS $1324+63$ has an overall spectral morphology similar to the T2 standard, particularly from the red optical to the blue slope of the $H$-band peak $(1.5 \mu \mathrm{m})$, at longer wavelengths it is considerbaly more red, with weaker $\mathrm{CH}_{4}$ absorption at $1.6 \mu \mathrm{m}$ and a brighter $K$-band peak $(2.1 \mu \mathrm{m})$. This motivates our classification of this source as peculiar. There are a handful of peculiar T dwarfs known, some of which are known to be binary (e.g., 2MASS J05185995-2828372; Cruz et al. 2004) or suspected to be affected by gravity effects (e.g., 2MASSI J0937347+293142; Burgasser et al. 2002, 2006a; Knapp et al. 2004). We consider both of these possibilities for 2MASS 1324+63.

To examine the binary hypothesis, we constructed a suite of synthetic binaries from L8-T8 spectral templates with equivalent 

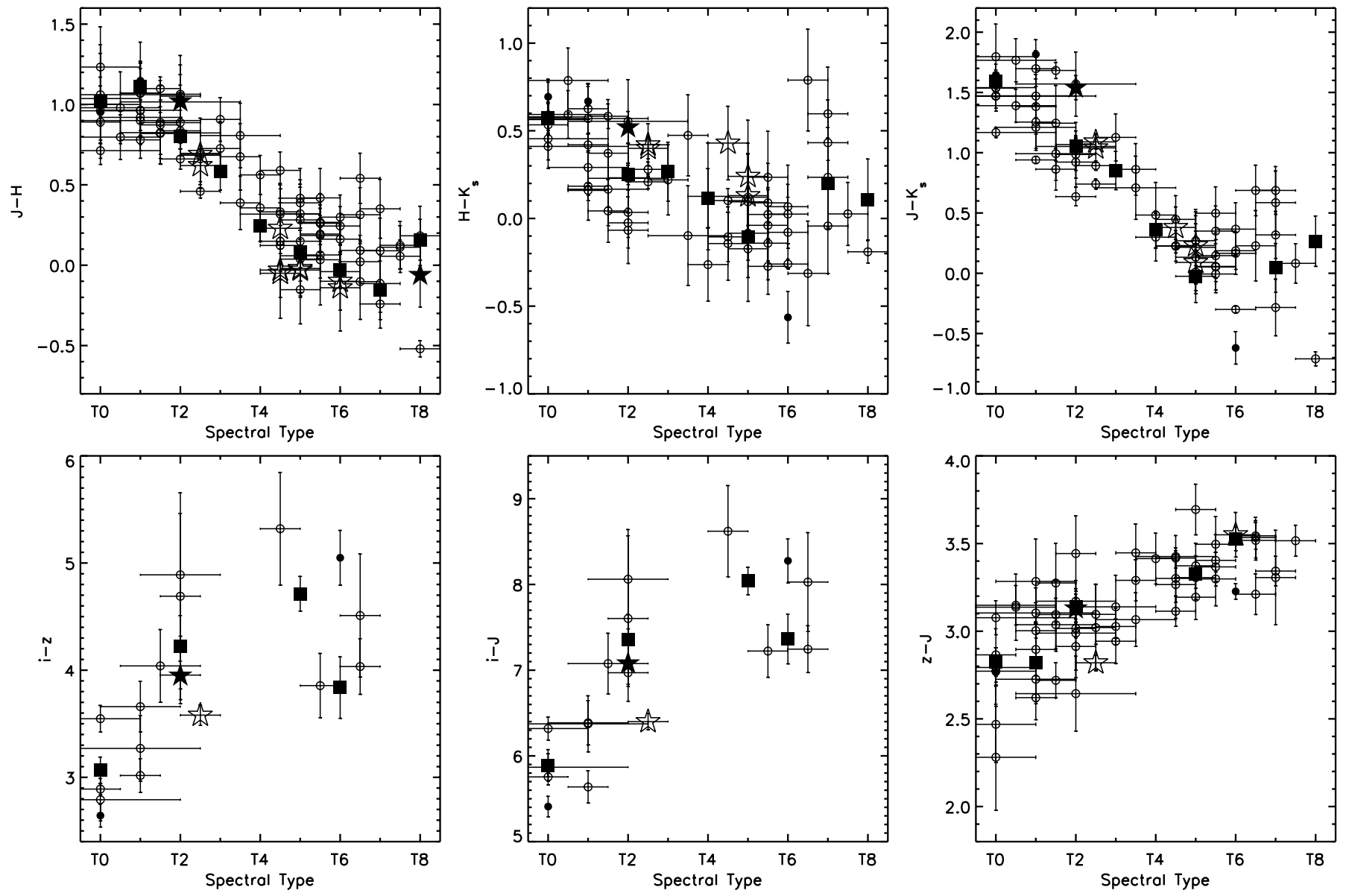

FIG. 5.-Far optical and NIR colors of known T dwarfs (open circles), known peculiar T dwarfs ( filled circles), T dwarf standards ( filled squares), new T dwarfs (open stars), and new peculiar T dwarfs ( filled stars) vs. NIR spectral type. All known T dwarfs shown have magnitude errors of less than 0.3 mag. The $i$ and $z$ magnitudes are from the SDSS (AB) catalog, and the $J, H$, and $K_{s}$ magnitudes are from the 2MASS catalog.

SpeX prism spectra. ${ }^{10}$ Our technique is similar to that used in Burgasser et al. (2005b, 2006c) and Reid et al. (2006). Our spectral templates include the primary NIR T dwarf spectral standards defined by Burgasser et al. (2006b) and the L dwarfs 2MASSW J1632291+190441 (L8; Kirkpatrick et al. 1999) and 2MASSW J0310599+164816 (L9; Kirkpatrick et al. 2000). We augmented the SpeX spectra from Burgasser et al. (2006b) with new spectra for SDSS J120747.17+024424.8 (T0; Hawley et al. 2002) and SDSSp J083717.22-000018.3 (T1; Leggett et al. 2000). Most of these sources are unresolved in high angular resolution imaging (Burgasser et al. 2006c, 2006b and refer-

${ }^{10}$ Synthetic spectra available on request. ences therein), with the exception of 2MASS 0310+16, which is a near-equal-brightness binary (Stumpf et al. 2005). The L9, $\mathrm{T} 0, \mathrm{~T} 3$, and $\mathrm{T} 7$ templates have yet to be imaged at high angular resolution.

Integrated light spectra for artificial binaries were constructed by first absolutely flux calibrating the $\mathrm{A}+\mathrm{B}$ spectral components. We computed a correction factor to scale the spectrum of each component such that it had the appropriate absolute $J$-band magnitude given by its spectral type. The correction factor is given by

$$
C_{J}=10^{-0.4 M_{J}} \frac{\int \lambda f_{\lambda}^{\mathrm{Vega}}(\lambda) T_{J}(\lambda) d \lambda}{\int \lambda f_{\lambda}^{\mathrm{obs}}(\lambda) T_{J}(\lambda) d \lambda}
$$

TABLE 6

Kinematic Properties of Four T Dwarfs with Multiepoch Measurements

\begin{tabular}{|c|c|c|c|c|c|c|}
\hline Object & Spectral Type & $\begin{array}{c}\mu \\
\left(\operatorname{arcsec} \mathrm{yr}^{-1}\right)\end{array}$ & $\begin{array}{c}\theta \\
(\mathrm{deg})\end{array}$ & $\begin{array}{c}\Delta t \\
(\mathrm{yr})\end{array}$ & $\begin{array}{l}\text { Distance } \\
\quad(\mathrm{pc})\end{array}$ & $\begin{array}{c}V_{\tan ^{\mathrm{a}}} \\
\left(\mathrm{km} \mathrm{s}^{-1}\right)\end{array}$ \\
\hline 2 MASS $1106+27^{\mathrm{b}} .$. & $\mathrm{T} 2.5$ & $0.57 \pm 0.08$ & $216.6 \pm 0.3$ & 2.13 & $15.5 \pm 1.2$ & $42 \pm 2$ \\
\hline 2MASS $1324+63^{\mathrm{b}} \ldots \ldots \ldots \ldots \ldots \ldots \ldots$ & T2:pec & $0.43 \pm 0.08$ & $232.8 \pm 0.3$ & 1.04 & $21.8 \pm 1.7$ & $45 \pm 3$ \\
\hline 2MASS $1404-31^{\mathrm{b}} \ldots \ldots \ldots \ldots \ldots$ & $\mathrm{T} 2.5$ & $0.35 \pm 0.03$ & $275.3 \pm 0.2$ & 2.55 & $22.0 \pm 1.7$ & $36 \pm 2$ \\
\hline 2MASS $1615+13^{\mathrm{c}} \ldots \ldots \ldots \ldots \ldots \ldots$ & T6 & $0.48 \pm 0.05$ & $139.0 \pm 0.2$ & 6.22 & $29.0 \pm 2.3$ & $66 \pm 4$ \\
\hline
\end{tabular}

a $V_{\tan }=4.74 \times D \times \mu^{\prime}$ and $\sigma_{V_{\text {tan }}}=\left[4.74\left(\mu^{\prime} \sigma_{D}^{2}+D \sigma_{\mu^{\prime}}^{2}\right)\right]^{1 / 2}$, where $\mu^{\prime}=\mu \times 1 \mathrm{yr}(\operatorname{arcsec})$.

${ }^{\mathrm{b}}$ Both epochs are from the 2MASS Working Database.

${ }^{c}$ The first epoch is from the 2MASS Point Source Catalog, and the second epoch is from the SDSS Catalog. 
TABLE 7

Synthetic Spectral Indices ${ }^{a}$

\begin{tabular}{|c|c|c|c|c|c|c|c|c|c|}
\hline \multirow[b]{2}{*}{$\begin{array}{c}\text { SPeCtRaL TYPE OF A+B } \\
\text { (1) }\end{array}$} & \multirow[b]{2}{*}{$\begin{array}{l}\mathrm{H}_{2} \mathrm{O}-J \\
\quad(2)\end{array}$} & \multirow[b]{2}{*}{$\begin{array}{l}\mathrm{CH}_{4}-J \\
\text { (3) }\end{array}$} & \multirow[b]{2}{*}{$\begin{array}{l}\mathrm{H}_{2} \mathrm{O}-H \\
\quad(4)\end{array}$} & \multirow[b]{2}{*}{$\begin{array}{c}\mathrm{CH}_{4}-\mathrm{H} \\
(5)\end{array}$} & \multirow[b]{2}{*}{$\begin{array}{l}\mathrm{CH}_{4}-K \\
(6)\end{array}$} & \multirow[b]{2}{*}{$\begin{array}{l}\mathrm{H} 2 \mathrm{O}-K \\
(7)\end{array}$} & \multirow[b]{2}{*}{$\begin{array}{l}K / J \\
(8)\end{array}$} & \multicolumn{2}{|c|}{ Derived NIR Spectral Type } \\
\hline & & & & & & & & $\begin{array}{l}\text { Direct }{ }^{\mathrm{b}, \mathrm{c}} \\
\text { (9) }\end{array}$ & $\begin{array}{l}\text { Ind } 1^{\mathrm{b}, \mathrm{d}, \mathrm{e}} \\
(10)\end{array}$ \\
\hline \multicolumn{10}{|c|}{ L8 Primary } \\
\hline 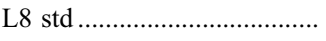 & 0.706 (L8) & 0.735 (L8) & 0.705 (L8) & 1.077 (L8) & 0.881 (L8) & 0.696 & 0.743 & L8 & L8 \\
\hline 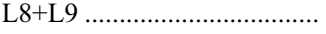 & 0.666 (L8.5) & $0.705(\mathrm{~L} 8.5)$ & $0.661(\mathrm{~L} 8.5)$ & $1.085(<\mathrm{L} 8)$ & $0.887(<\mathrm{L} 8)$ & 0.697 & 0.688 & L8.5 & L8.5 \\
\hline L8+T0 & 0.657 (L8.5) & 0.685 (L9) & 0.655 (L8.5) & 1.012 (L9.5) & 0.840 (L9.5) & 0.689 & 0.570 & L9 & L9 \\
\hline 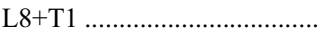 & 0.630 (L9) & 0.683 (L9) & 0.625 (L9) & 1.023 (L9.5) & 0.807 ( T0) & 0.660 & 0.525 & L9.5 & L9.5 \\
\hline 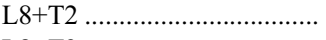 & $0.550(\mathrm{~T} 1.5)$ & $0.631(\mathrm{~T} 1.5)$ & $0.566(\mathrm{~T} 1)$ & $0.981(\mathrm{~T} 1)$ & $0.732(\mathrm{~T} 1)$ & 0.624 & 0.490 & T0 & $\mathrm{T} 1$ \\
\hline L8+T3 & $0.502(\mathrm{~T} 1.5)$ & $0.582(\mathrm{~T} 2)$ & $0.554(\mathrm{~T} 1)$ & $0.862(\mathrm{~T} 2.5)$ & $0.712(\mathrm{~T} 1)$ & 0.618 & 0.413 & T1 & $\mathrm{T} 1.5$ \\
\hline 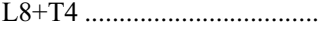 & $0.478(\mathrm{~T} 2)$ & $0.580(\mathrm{~T} 2)$ & $0.527(\mathrm{~T} 1.5)$ & $0.798(\mathrm{~T} 2.5)$ & $0.652(\mathrm{~T} 1.5)$ & 0.611 & 0.404 & T1.5: & T2 \\
\hline L8+T5 & 0.407 (T3) & $0.492(\mathrm{~T} 4)$ & $0.542(\mathrm{~T} 1)$ & $0.767(\mathrm{~T} 2.5)$ & $0.705(\mathrm{~T} 1)$ & 0.631 & 0.367 & T2: & T2.5: \\
\hline L8+T6 & $0.404(\mathrm{~T} 3)$ & $0.526(\mathrm{~T} 3)$ & $0.554(\mathrm{~T} 1)$ & $0.802(\mathrm{~T} 2.5)$ & $0.750(\mathrm{~T} 0.5)$ & 0.644 & 0.418 & T2: & T2: \\
\hline L8+T7 & $0.447(\mathrm{~T} 2.5)$ & $0.528(\mathrm{~T} 3)$ & $0.585(\mathrm{~T} 0.5)$ & $0.853(\mathrm{~T} 2.5)$ & $0.775(\mathrm{~T} 0)$ & 0.648 & 0.504 & T1: & T1.5: \\
\hline 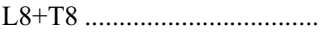 & $0.522(\mathrm{~T} 1.5)$ & $0.583(\mathrm{~T} 2)$ & 0.620 (L9) & $0.919(\mathrm{~T} 2)$ & 0.833 (L9.5) & 0.672 & 0.577 & T0: & T1: \\
\hline \multicolumn{10}{|c|}{ L9 Primary } \\
\hline L9 $\operatorname{std} \ldots$ & 0.631 (L9) & 0.681 (L9) & 0.621 (L9) & 1.092 (L9) & 0.894 (L9) & 0.698 & 0.640 & L9 & L9 \\
\hline $\mathrm{L} 9+\mathrm{T} 0 \ldots \ldots$. & 0.626 (L9.5) & 0.664 (L9.5) & 0.616 (L9.5) & 1.022 (L9.5) & 0.847 (L9.5) & 0.690 & 0.533 & L9.5 & L9.5 \\
\hline L9+T1 & $0.604(\mathrm{~T} 0.5)$ & 0.664 (L9.5) & $0.589(\mathrm{~T} 0.5)$ & 1.032 (L9.5) & $0.815(\mathrm{~T} 0)$ & 0.661 & 0.494 & L9.5 & T0 \\
\hline L9+T2 & $0.531(\mathrm{~T} 1.5)$ & $0.618(\mathrm{~T} 1.5)$ & $0.535(\mathrm{~T} 1.5)$ & 0.990 (L9.5) & $0.740(\mathrm{~T} 1)$ & 0.625 & 0.464 & $\mathrm{~T} 0.5$ & $\mathrm{~T} 1$ \\
\hline 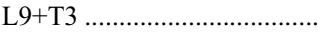 & $0.486(\mathrm{~T} 2)$ & $0.571(\mathrm{~T} 2)$ & $0.523(\mathrm{~T} 1.5)$ & $0.875(\mathrm{~T} 2)$ & $0.721(\mathrm{~T} 1)$ & 0.619 & 0.392 & $\mathrm{~T} 1.5$ & $\mathrm{~T} 1.5$ \\
\hline L9+T4 & $0.463(\mathrm{~T} 2)$ & $0.567(\mathrm{~T} 2)$ & $0.495(\mathrm{~T} 2)$ & $0.815(\mathrm{~T} 2.5)$ & $0.662(\mathrm{~T} 1.5)$ & 0.613 & 0.383 & $\mathrm{~T} 2$ & $\mathrm{~T} 2$ \\
\hline 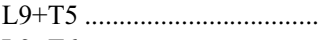 & $0.394(\mathrm{~T} 3.5)$ & $0.484(\mathrm{~T} 4)$ & $0.502(\mathrm{~T} 1.5)$ & $0.789(\mathrm{~T} 2.5)$ & $0.717(\mathrm{~T} 1)$ & 0.633 & 0.345 & T2.5: & T2.5: \\
\hline 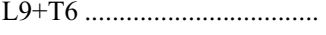 & $0.387(\mathrm{~T} 3.5)$ & 0.514 (T3) & $0.506(\mathrm{~T} 1.5)$ & $0.826(\mathrm{~T} 2.5)$ & $0.762(\mathrm{~T} 0.5)$ & 0.646 & 0.387 & T2.5: & T2: \\
\hline 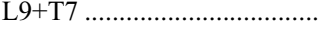 & $0.422(\mathrm{~T} 3)$ & $0.511(\mathrm{~T} 3.5)$ & $0.528(\mathrm{~T} 1.5)$ & $0.878(\mathrm{~T} 2)$ & $0.788(\mathrm{~T} 0)$ & 0.650 & 0.459 & T2: & T2: \\
\hline L9+T8 & $0.485(\mathrm{~T} 2)$ & $0.558(\mathrm{~T} 2.5)$ & $0.555(\mathrm{~T} 1)$ & $0.942(\mathrm{~T} 1.5)$ & 0.846 (L9.5) & 0.675 & 0.515 & T1: & T1.5: \\
\hline \multicolumn{10}{|c|}{ T0 Primary } \\
\hline 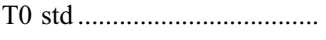 & $0.621(\mathrm{~T} 0)$ & $0.651(\mathrm{~T} 0)$ & $0.612(\mathrm{~T} 0)$ & $0.955(\mathrm{~T} 0)$ & $0.790(\mathrm{~T} 0)$ & 0.680 & 0.442 & T0 & T0 \\
\hline 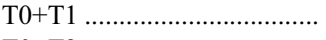 & $0.602(\mathrm{~T} 0.5)$ & $0.651(\mathrm{~T} 0)$ & $0.585(\mathrm{~T} 0.5)$ & $0.969(\mathrm{~T} 0)$ & $0.755(\mathrm{~T} 0.5)$ & 0.648 & 0.414 & $\mathrm{~T} 0.5$ & T0.5 \\
\hline T0+T2 & $0.533(\mathrm{~T} 1.5)$ & $0.610(\mathrm{~T} 1.5)$ & $0.533(\mathrm{~T} 1.5)$ & $0.933(\mathrm{~T} 1.5)$ & $0.677(\mathrm{~T} 1.5)$ & 0.610 & 0.396 & $\mathrm{~T} 1$ & $\mathrm{~T} 1.5$ \\
\hline 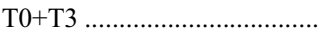 & $0.491(\mathrm{~T} 2)$ & $0.566(\mathrm{~T} 2.5)$ & $0.521(\mathrm{~T} 1.5)$ & $0.820(\mathrm{~T} 2.5)$ & 0.647 (T1.5) & 0.601 & 0.332 & $\mathrm{~T} 2$ & $\mathrm{~T} 2$ \\
\hline 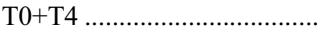 & $0.469(\mathrm{~T} 2)$ & $0.562(\mathrm{~T} 2.5)$ & $0.493(\mathrm{~T} 2)$ & $0.756(\mathrm{~T} 3)$ & $0.574(\mathrm{~T} 2)$ & 0.592 & 0.320 & T2.5: & $\mathrm{T} 2.5$ \\
\hline T0+T5 & $0.405(\mathrm{~T} 3)$ & $0.483(\mathrm{~T} 4)$ & $0.499(\mathrm{~T} 1.5)$ & $0.718(\mathrm{~T} 3)$ & $0.615(\mathrm{~T} 2)$ & 0.610 & 0.279 & $\mathrm{~T} 3$ & $\mathrm{~T} 2.5$ \\
\hline 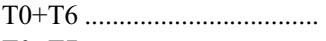 & $0.402(\mathrm{~T} 3)$ & $0.511(\mathrm{~T} 3.5)$ & $0.504(\mathrm{~T} 1.5)$ & $0.742(\mathrm{~T} 3)$ & $0.656(\mathrm{~T} 1.5)$ & 0.623 & 0.303 & T3: & $\mathrm{T} 2.5$ \\
\hline 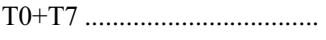 & $0.436(\mathrm{~T} 2.5)$ & $0.508(\mathrm{~T} 4)$ & $0.524(\mathrm{~T} 1.5)$ & $0.779(\mathrm{~T} 2.5)$ & $0.679(\mathrm{~T} 1.5)$ & 0.626 & 0.347 & T2: & T2.5: \\
\hline 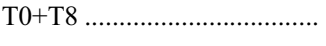 & $0.495(\mathrm{~T} 2)$ & $0.548(\mathrm{~T} 2.5)$ & $0.549(\mathrm{~T} 1)$ & $0.831(\mathrm{~T} 2.5)$ & $0.739(\mathrm{~T} 1)$ & 0.653 & 0.376 & T1: & $\mathrm{T} 2$ \\
\hline \multicolumn{10}{|c|}{ T1 Primary } \\
\hline 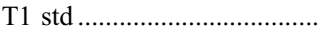 & $0.584(\mathrm{~T} 1)$ & $0.653(\mathrm{~T} 1)$ & $0.563(\mathrm{~T} 1)$ & $0.981(\mathrm{~T} 1)$ & $0.724(\mathrm{~T} 1)$ & 0.620 & 0.391 & $\mathrm{~T} 1$ & $\mathrm{~T} 1$ \\
\hline $\mathrm{T} 1+\mathrm{T} 2$ & $0.523(\mathrm{~T} 1.5)$ & $0.613(\mathrm{~T} 1.5)$ & $0.515(\mathrm{~T} 1.5)$ & $0.947(\mathrm{~T} 1.5)$ & $0.650(\mathrm{~T} 1.5)$ & 0.584 & 0.377 & $\mathrm{~T} 1.5$ & $\mathrm{~T} 1.5$ \\
\hline 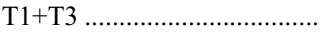 & $0.485(\mathrm{~T} 2)$ & $0.573(\mathrm{~T} 2)$ & $0.504(\mathrm{~T} 1.5)$ & $0.840(\mathrm{~T} 2.5)$ & $0.616(\mathrm{~T} 2)$ & 0.572 & 0.318 & $\mathrm{~T} 2$ & $\mathrm{~T} 2$ \\
\hline 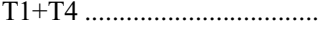 & $0.464(\mathrm{~T} 2)$ & $0.570(\mathrm{~T} 2)$ & $0.476(\mathrm{~T} 2)$ & $0.782(\mathrm{~T} 2.5)$ & $0.545(\mathrm{~T} 2.5)$ & 0.561 & 0.307 & T2.5 & T2 \\
\hline 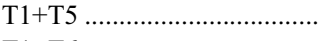 & $0.406(\mathrm{~T} 3)$ & 0.499 (T4) & $0.478(\mathrm{~T} 2)$ & $0.752(\mathrm{~T} 3)$ & $0.576(\mathrm{~T} 2)$ & 0.571 & 0.267 & $\mathrm{~T} 3$ & T3 \\
\hline 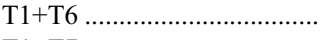 & $0.402(\mathrm{~T} 3)$ & $0.526(\mathrm{~T} 3)$ & $0.478(\mathrm{~T} 2)$ & $0.778(\mathrm{~T} 2.5)$ & $0.610(\mathrm{~T} 2)$ & 0.578 & 0.287 & $\mathrm{~T} 3$ & $\mathrm{~T} 2.5$ \\
\hline 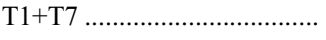 & $0.433(\mathrm{~T} 2.5)$ & $0.528(\mathrm{~T} 3)$ & $0.494(\mathrm{~T} 2)$ & $0.817(\mathrm{~T} 2.5)$ & $0.629(\mathrm{~T} 1.5)$ & 0.578 & 0.323 & $\mathrm{~T} 2.5$ & $\mathrm{~T} 2.5$ \\
\hline T1+T8 & $0.483(\mathrm{~T} 2)$ & $0.565(\mathrm{~T} 2.5)$ & $0.513(\mathrm{~T} 1.5)$ & $0.867(\mathrm{~T} 2.5)$ & $0.680(\mathrm{~T} 1.5)$ & 0.599 & 0.343 & $\mathrm{~T} 2$ & $\mathrm{~T} 2$ \\
\hline
\end{tabular}


TABLE 7-Continued

\begin{tabular}{|c|c|c|c|c|c|c|c|c|c|}
\hline \multirow[b]{2}{*}{$\begin{array}{c}\text { Spectral Type of A+B } \\
\text { (1) }\end{array}$} & \multirow[b]{2}{*}{$\begin{array}{l}\mathrm{H}_{2} \mathrm{O}-J \\
\quad(2)\end{array}$} & \multirow[b]{2}{*}{$\begin{array}{l}\mathrm{CH}_{4}-J \\
\text { (3) }\end{array}$} & \multirow[b]{2}{*}{$\begin{array}{l}\mathrm{H}_{2} \mathrm{O}-H \\
\quad(4)\end{array}$} & \multirow[b]{2}{*}{$\begin{array}{l}\mathrm{CH}_{4}-\mathrm{H} \\
(5)\end{array}$} & \multirow[b]{2}{*}{$\begin{array}{l}\mathrm{CH}_{4}-\mathrm{K} \\
(6)\end{array}$} & \multirow[b]{2}{*}{$\begin{array}{l}\mathrm{H} 2 \mathrm{O}-K \\
\text { (7) }\end{array}$} & \multirow[b]{2}{*}{$\begin{array}{c}K / J \\
(8)\end{array}$} & \multicolumn{2}{|c|}{ Derived NIR Spectral Type } \\
\hline & & & & & & & & $\begin{array}{l}\text { Direct }^{\mathrm{b}, \mathrm{c}} \\
\quad(9)\end{array}$ & $\begin{array}{l}\text { Ind } 1^{\mathrm{b}, \mathrm{d}, \mathrm{e}} \\
(10)\end{array}$ \\
\hline \multicolumn{10}{|c|}{ T2 Primary } \\
\hline $\mathrm{T} 2 \mathrm{std}$ & $0.474(\mathrm{~T} 2)$ & $0.583(\mathrm{~T} 2)$ & $0.474(\mathrm{~T} 2)$ & $0.917(\mathrm{~T} 2)$ & $0.585(\mathrm{~T} 2)$ & 0.552 & 0.365 & $\mathrm{~T} 2$ & $\mathrm{~T} 2$ \\
\hline T2+T3 & $0.442(\mathrm{~T} 2.5)$ & $0.547(\mathrm{~T} 2.5)$ & $0.463(\mathrm{~T} 2.5)$ & $0.818(\mathrm{~T} 2.5)$ & $0.546(\mathrm{~T} 2.5)$ & 0.537 & 0.312 & $\mathrm{~T} 2.5$ & $\mathrm{~T} 2.5$ \\
\hline $\mathrm{T} 2+\mathrm{T} 4 \mathrm{\ldots}$ & $0.422(\mathrm{~T} 3)$ & $0.544(\mathrm{~T} 2.5)$ & $0.435(\mathrm{~T} 3.5)$ & 0.763 (T3) & $0.474(\mathrm{~T} 3)$ & 0.524 & 0.302 & $\mathrm{~T} 3$ & $\mathrm{~T} 3$ \\
\hline $\mathrm{T} 2+\mathrm{T} 5$ & $0.365(\mathrm{~T} 4)$ & $0.477(\mathrm{~T} 4)$ & $0.429(\mathrm{~T} 3.5)$ & $0.732(\mathrm{~T} 3)$ & $0.486(\mathrm{~T} 3)$ & 0.525 & 0.266 & $\mathrm{~T} 3$ & $\mathrm{~T} 3.5$ \\
\hline $\mathrm{T} 2+\mathrm{T} 6$ & $0.356(\mathrm{~T} 4)$ & $0.498(\mathrm{~T} 4)$ & $0.423(\mathrm{~T} 3.5)$ & $0.754(\mathrm{~T} 3)$ & $0.507(\mathrm{~T} 3)$ & 0.527 & 0.283 & $\mathrm{~T} 3$ & $\mathrm{~T} 3.5$ \\
\hline $\mathrm{T} 2+\mathrm{T} 7$ & $0.373(\mathrm{~T} 4)$ & $0.494(\mathrm{~T} 4)$ & $0.429(\mathrm{~T} 3.5)$ & $0.785(\mathrm{~T} 2.5)$ & $0.518(\mathrm{~T} 3)$ & 0.524 & 0.313 & $\mathrm{~T} 3$ & $\mathrm{~T} 3.5$ \\
\hline $\mathrm{T} 2+\mathrm{T} 8$ & $0.407(\mathrm{~T} 3)$ & $0.521(\mathrm{~T} 3)$ & $0.441(\mathrm{~T} 3)$ & $0.825(\mathrm{~T} 2.5)$ & $0.554(\mathrm{~T} 2.5)$ & 0.537 & 0.329 & $\mathrm{~T} 2.5$ & $\mathrm{~T} 3$ \\
\hline \multicolumn{10}{|c|}{ T3 Primary } \\
\hline 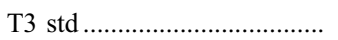 & $0.413(\mathrm{~T} 3)$ & $0.516(\mathrm{~T} 3)$ & $0.453(\mathrm{~T} 3)$ & $0.717(\mathrm{~T} 3)$ & 0.496 (T3) & 0.519 & 0.264 & $\mathrm{~T} 3$ & $\mathrm{~T} 3$ \\
\hline 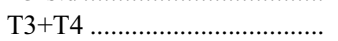 & $0.392(\mathrm{~T} 3.5)$ & $0.510(\mathrm{~T} 3.5)$ & $0.423(\mathrm{~T} 3.5)$ & $0.654(\mathrm{~T} 3.5)$ & $0.408(\mathrm{~T} 3.5)$ & 0.503 & 0.252 & $\mathrm{~T} 3.5$ & $\mathrm{~T} 3.5$ \\
\hline 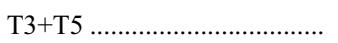 & $0.337(\mathrm{~T} 4)$ & $0.445(\mathrm{~T} 4.5)$ & $0.414(\mathrm{~T} 3.5)$ & $0.602(\mathrm{~T} 4)$ & $0.405(\mathrm{~T} 3.5)$ & 0.497 & 0.215 & $\mathrm{~T} 4$ & $\mathrm{~T} 4$ \\
\hline T3+T6 & $0.323(\mathrm{~T} 4.5)$ & $0.459(\mathrm{~T} 4.5)$ & $0.406(\mathrm{~T} 3.5)$ & $0.606(\mathrm{~T} 4)$ & $0.420(\mathrm{~T} 3.5)$ & 0.497 & 0.222 & $\mathrm{~T} 4$ & $\mathrm{~T} 4$ \\
\hline $\mathrm{T} 3+\mathrm{T} 7 \ldots \ldots \ldots \ldots$ & $0.334(\mathrm{~T} 4.5)$ & $0.449(\mathrm{~T} 4.5)$ & $0.411(\mathrm{~T} 3.5)$ & $0.619(\mathrm{~T} 3.5)$ & $0.427(\mathrm{~T} 3.5)$ & 0.490 & 0.240 & $\mathrm{~T} 4$ & $\mathrm{~T} 4$ \\
\hline $\mathrm{T} 3+\mathrm{T} 8$ & $0.359(\mathrm{~T} 4)$ & $0.468(\mathrm{~T} 4.5)$ & $0.422(\mathrm{~T} 3.5)$ & $0.647(\mathrm{~T} 3.5)$ & $0.464(\mathrm{~T} 3)$ & 0.503 & 0.245 & $\mathrm{~T} 3.5$ & $\mathrm{~T} 3.5$ \\
\hline \multicolumn{10}{|c|}{ T4 Primary } \\
\hline 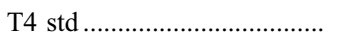 & $0.369(\mathrm{~T} 4)$ & $0.506(\mathrm{~T} 4)$ & $0.389(\mathrm{~T} 4)$ & $0.581(\mathrm{~T} 4)$ & $0.305(\mathrm{~T} 4)$ & 0.482 & 0.239 & $\mathrm{~T} 4$ & $\mathrm{~T} 4$ \\
\hline $\mathrm{T} 4+\mathrm{T} 5$ & $0.310(\mathrm{~T} 4.5)$ & $0.437(\mathrm{~T} 4.5)$ & $0.372(\mathrm{~T} 4.5)$ & $0.508(\mathrm{~T} 4.5)$ & $0.269(\mathrm{~T} 4.5)$ & 0.471 & 0.199 & $\mathrm{~T} 4.5$ & $\mathrm{~T} 4.5$ \\
\hline $\mathrm{T} 4+\mathrm{T} 6$ & $0.291(\mathrm{~T} 4.5)$ & $0.450(\mathrm{~T} 4.5)$ & $0.356(\mathrm{~T} 4.5)$ & $0.498(\mathrm{~T} 4.5)$ & $0.266(\mathrm{~T} 4.5)$ & 0.466 & 0.204 & $\mathrm{~T} 4.5$ & $\mathrm{~T} 4.5$ \\
\hline $\mathrm{T} 4+\mathrm{T} 7$ & $0.297(\mathrm{~T} 4.5)$ & $0.438(\mathrm{~T} 4.5)$ & $0.355(\mathrm{~T} 5)$ & $0.499(\mathrm{~T} 4.5)$ & $0.260(\mathrm{~T} 4.5)$ & 0.455 & 0.220 & $\mathrm{~T} 4.5$ & $\mathrm{~T} 4.5$ \\
\hline $\mathrm{T} 4+\mathrm{T} 8$ & $0.319(\mathrm{~T} 4.5)$ & $0.456(\mathrm{~T} 4.5)$ & $0.362(\mathrm{~T} 4.5)$ & $0.518(\mathrm{~T} 4.5)$ & $0.283(\mathrm{~T} 4)$ & 0.467 & 0.223 & $\mathrm{~T} 4$ & $\mathrm{~T} 4.5$ \\
\hline \multicolumn{10}{|c|}{ T5 Primary } \\
\hline 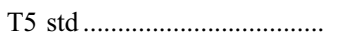 & $0.240(\mathrm{~T} 5)$ & $0.356(\mathrm{~T} 5)$ & $0.345(\mathrm{~T} 5)$ & $0.393(\mathrm{~T} 5)$ & $0.200(\mathrm{~T} 5)$ & 0.450 & 0.151 & T5 & $\mathrm{T} 5$ \\
\hline $\mathrm{T} 5+\mathrm{T} 6$ & $0.205(\mathrm{~T} 5.5)$ & $0.355(\mathrm{~T} 5.5)$ & $0.319(\mathrm{~T} 5.5)$ & $0.357(\mathrm{~T} 5.5)$ & $0.180(\mathrm{~T} 5.5)$ & 0.438 & 0.148 & T5.5 & T5.5 \\
\hline $\mathrm{T} 5+\mathrm{T} 7$ & $0.195(\mathrm{~T} 5.5)$ & $0.322(\mathrm{~T} 6.5)$ & $0.311(\mathrm{~T} 5.5)$ & $0.332(\mathrm{~T} 5.5)$ & 0.159 (T6) & 0.417 & 0.155 & T5.5 & T6 \\
\hline $\mathrm{T} 5+\mathrm{T} 8$ & $0.205(\mathrm{~T} 5.5)$ & $0.325(\mathrm{~T} 6.5)$ & $0.315(\mathrm{~T} 5.5)$ & $0.338(\mathrm{~T} 5.5)$ & $0.177(\mathrm{~T} 5.5)$ & 0.428 & 0.148 & T5.5 & $\mathrm{T} 5.5$ \\
\hline \multicolumn{10}{|c|}{ T6 Primary } \\
\hline 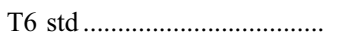 & $0.154(\mathrm{~T} 6)$ & $0.354(\mathrm{~T} 6)$ & $0.280(\mathrm{~T} 6)$ & $0.301(\mathrm{~T} 6)$ & $0.149(\mathrm{~T} 6)$ & 0.418 & 0.142 & $\mathrm{~T} 6$ & $\mathrm{~T} 6$ \\
\hline 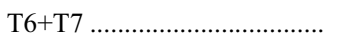 & $0.129(\mathrm{~T} 6.5)$ & $0.311(\mathrm{~T} 6.5)$ & $0.259(\mathrm{~T} 6.5)$ & $0.256(\mathrm{~T} 6.5)$ & $0.114(\mathrm{~T} 6.5)$ & 0.387 & 0.150 & $\mathrm{~T} 6.5$ & T6.5 \\
\hline T6+T8 ……............................ & $0.127(\mathrm{~T} 6.5)$ & $0.313(\mathrm{~T} 6.5)$ & $0.255(\mathrm{~T} 6.5)$ & $0.250(\mathrm{~T} 6.5)$ & $0.127(\mathrm{~T} 6.5)$ & 0.394 & 0.140 & $\mathrm{~T} 6.5$ & T6.5 \\
\hline \multicolumn{10}{|c|}{ T7 Primary } \\
\hline 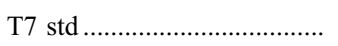 & $0.085(\mathrm{~T} 7)$ & $0.238(\mathrm{~T} 7)$ & $0.224(\mathrm{~T} 7)$ & $0.181(\mathrm{~T} 7)$ & $0.062(\mathrm{~T} 7)$ & 0.340 & 0.164 & $\mathrm{~T} 7$ & $\mathrm{~T} 7$ \\
\hline T7+T8 & $0.070(\mathrm{~T} 7.5)$ & $0.219(\mathrm{~T} 7.5)$ & $0.209(\mathrm{~T} 7.5)$ & $0.153(\mathrm{~T} 7.5)$ & $0.059(\mathrm{~T} 7)$ & 0.332 & 0.152 & $\mathrm{~T} 7.5$ & $\mathrm{~T} 7.5$ \\
\hline \multicolumn{10}{|c|}{ T8 Standard } \\
\hline 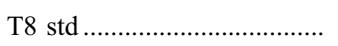 & $0.041(\mathrm{~T} 8)$ & $0.182(\mathrm{~T} 8)$ & $0.183(\mathrm{~T} 8)$ & $0.104(\mathrm{~T} 8)$ & $0.050(\mathrm{~T} 8)$ & 0.311 & 0.131 & $\mathrm{~T} 8$ & $\mathrm{~T} 8$ \\
\hline
\end{tabular}



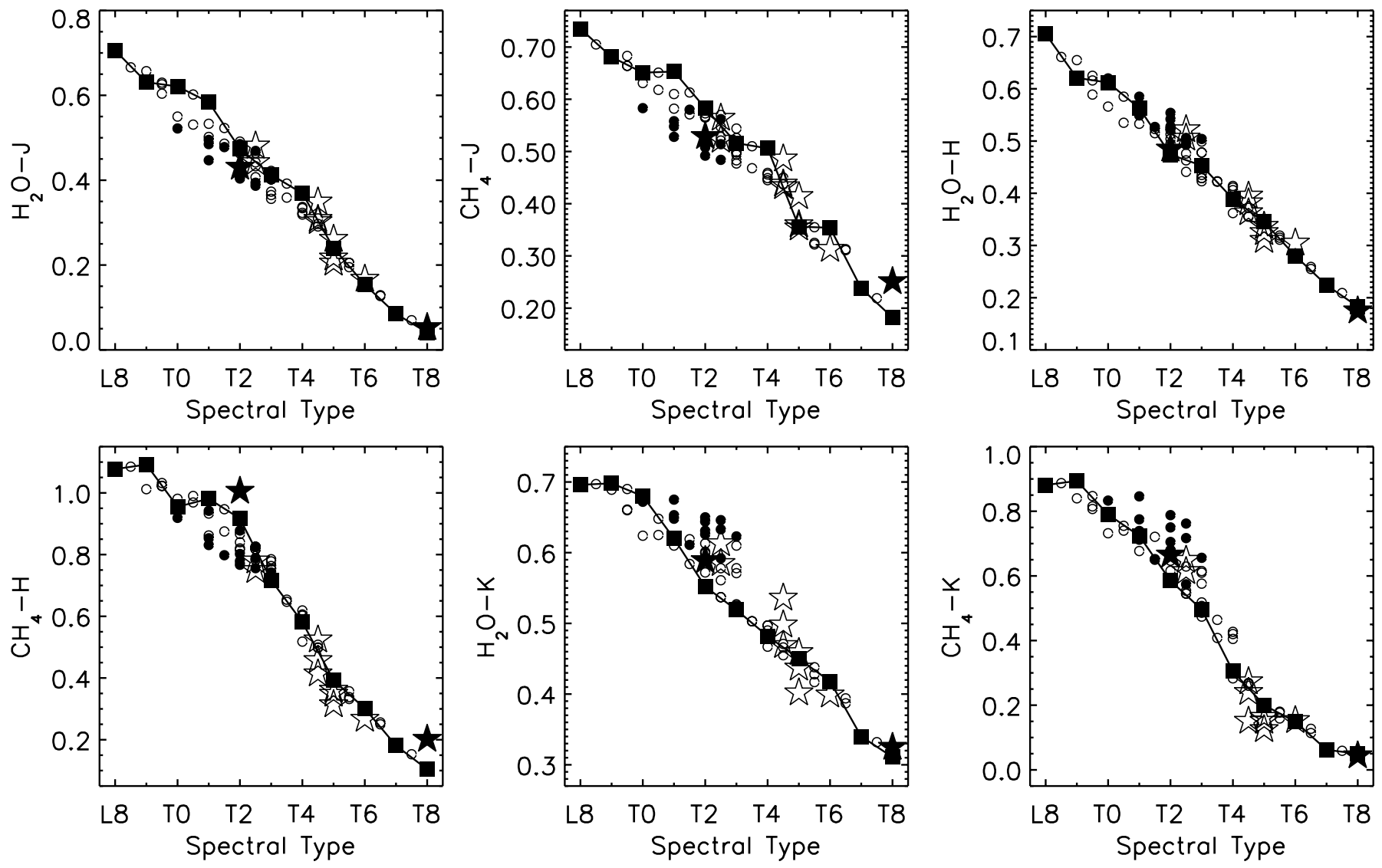

FIG. 6.- Spectral index values of $\mathrm{H}_{2} \mathrm{O}$ and $\mathrm{CH}_{4}$ features vs. NIR spectral type (determined by direct comparison) as defined by Burgasser et al. (2006b). Filled squares represent the late-type $\mathrm{L}$ and $\mathrm{T}$ dwarfs we used to create the synthetic spectra, with a solid line connecting them. Synthetic spectra are represented by circles, with peculiar or uncertain types denoted by filled circles. The 11 new T dwarfs reported here are indicated by stars, with peculiar sources denoted by filled stars.

where both the $J$-band Vega spectrum, ${ }^{11} f_{\lambda}^{\text {Vega }}$, and the 2 MASS $J$-band transmission profile, ${ }^{12} T_{J}(\lambda)$, have been interpolated onto the wave scale of each observed component. The absolute $J$-band magnitude, $M_{J}$, was computed from the Liu et al. (2006) spectral type versus absolute magnitude relation, excluding known binaries. We calculated and multiplied this correction factor for both $\mathrm{A}+\mathrm{B}$ components. Then we interpolated the wavelength scale of the B component onto the wavelength scale of the A component and added the two fluxes for the combined synthetic spectrum. For quantitative reference, we calculated $\mathrm{H}_{2} \mathrm{O}$ and $\mathrm{CH}_{4}$ spectral indices for the entire suite (see cols. [2]-[6] in Table 7) but classify the synthetic spectra on overall morphology in comparison with spectral standards (see col. [9] of Table 7, "Direct"). The spectral type of each synthetic combination computed by the indices is shown in column (10) of Table 7 ("Ind 1"). These indices are shown graphically in Figure 6 for the NIR synthetic spectra, NIR T dwarf standards, and the 11 new T dwarfs.

The synthetic binary spectra were compared to the spectrum of 2MASS $1324+63$ visually. The four closest matches are shown in Figure 7. The best two matches are L9+T2 [T0.5] and L8+T5 [T2:]. The L9+T2 synthetic combination, while providing a fairly good match throughout most of the $H$ and $K_{s}$ bands and having comparable $\mathrm{H}_{2} \mathrm{O}$ absorption strength from 1.75 to $2.05 \mu \mathrm{m}$, has weaker $\mathrm{H}_{2} \mathrm{O}$ absorption from 1.3 to $1.5 \mu \mathrm{m}$, a weaker Y-band ( $\sim 0.93-1.15 \mu \mathrm{m}$; Hillenbrand et al. 2002) slope, and

${ }^{11}$ Downloadable from Sandy Leggett's site: ftp://ftp.jach.hawaii.edu/pub/ ukirt/sk1/filters/vega.obs.

${ }^{12}$ See http://spider.ipac.caltech.edu/staff/waw/2mass/opt_cal/jrsr.tbl.html. weaker $\mathrm{CH}_{4}$ and $\mathrm{H}_{2} \mathrm{O}$ absorption from $\sim 1.1$ to $1.2 \mu \mathrm{m}$. The L8+T5 synthetic combination, conversely, provides a good match in the $Y$ and $J$ bands but has overly strong absorption in the $H$ and $K$ bands, which are primarily shaped by $\mathrm{CH}_{4}$ absorption and CIA $\mathrm{H}_{2}$. While none of these matches are ideal, it should be noted that similar analysis for the resolved binary DENIS-P J225210.73-173013.4 also fails to provide a perfect fit, even though the source is known to be a binary (Reid et al. 2006). So unresolved multiplicity may nevertheless play a significant role.

Another possibility is that 2 MASS $1324+63$ is a young T dwarf $(<300 \mathrm{Myr})$, implying a lower mass, larger radius, and hence lower surface gravity (low-pressure photosphere). One of the primary spectral shapers in the $K$ band and, to a lesser extent, the $\mathrm{H}$ band is CIA $\mathrm{H}_{2}$. In a low-pressure atmosphere, the contribution of CIA $\mathrm{H}_{2}$ would decrease and the spectrum would become redder ( $J-K$ would increase). This effect has been noted for young L dwarfs (Kirkpatrick et al. 2006; J. D. Kirkpatrick et al. 2007 , in preparation). 2MASS $1324+63$ could also have a dustier photosphere than typical T2 dwarfs, possibly caused by a lower gravity environment retarding the precipitation of dust. To date, the youngest spectroscopically confirmed T dwarf ${ }^{13}$ is HN Peg B (Luhman et al. 2007), a T2.5 companion to HN Peg A (a G0 $\mathrm{V}$ star estimated to be $\sim 300 \mathrm{Myr}$ old). After $\sim 300 \mathrm{Myr}$, brown dwarf radii contract very little, differing by only $\sim 20 \%$ in radius

\footnotetext{
13 A candidate very low mass T dwarf in the $\sim 1$ Myr Orion cluster, S Ori 70 , has been proposed by Zapatero Osorio et al. (2002) and Martín \& Osorio (2003). However, this source remains controversial (Burgasser et al. 2004a).
} 


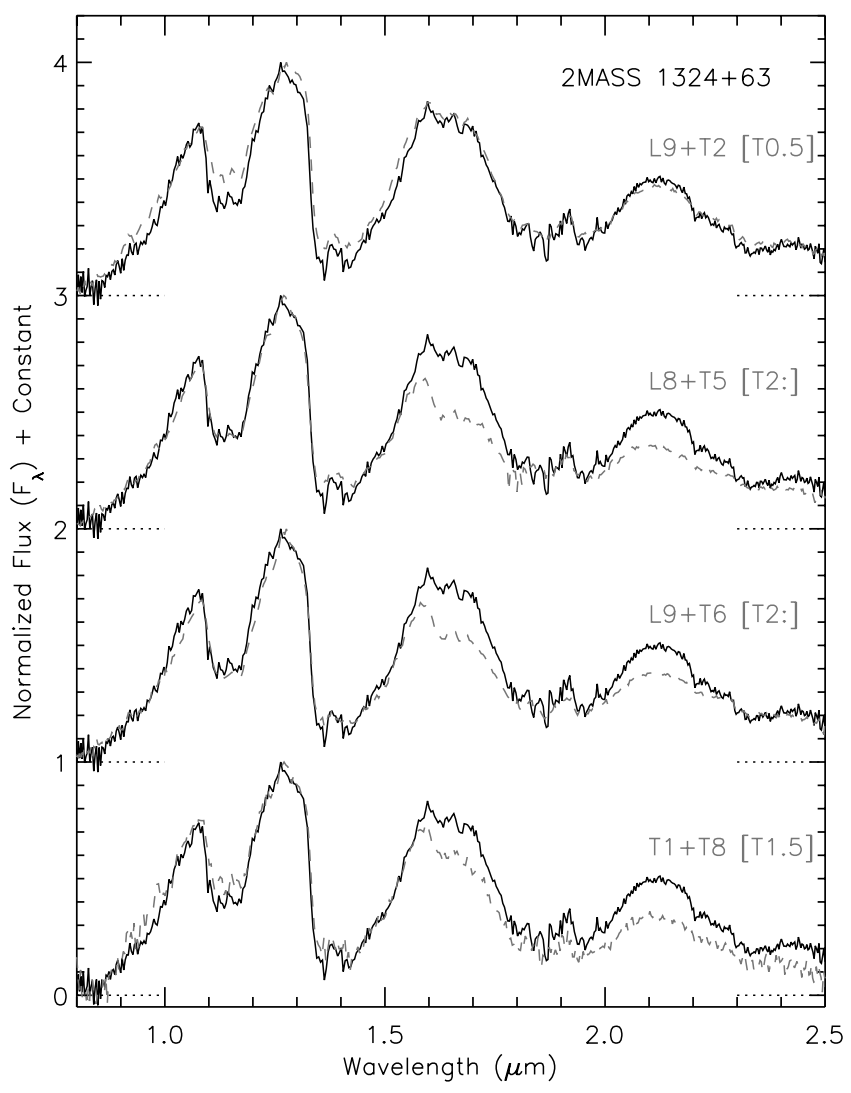

Fig. 7.- Spectral template fitting of 2MASS 1324+63 (black) overplotted with best synthetic spectral fits ( gray) determined visually. The A+B components are labeled, and the integrated light spectral type (determined by direct comparison) is shown in brackets. All spectra have been normalized at $1.27 \mu \mathrm{m}$ and are offset by integer units (zero levels are marked by dotted lines) for clarity. Of these four, the best fit is L9+T2 [T0.5].

from their much older 3 Gyr counterparts (Burrows et al. 1997). While this relatively young object is less massive than a $3 \mathrm{Gyr}$ T2.5 dwarf, HN Peg B shows no spectroscopic deviations from a typical field T2.5 dwarf (see Fig. 8 and Luhman et al. 2007).

2 MASS $1324+63$ is located in a region of sky completely unoccupied by any currently known young moving group. It could be a member of an unidentified young moving group or it could be older (>100 Myr) than most moving groups (either a castoff or from a dispersed group). Its tangential velocity ( $V_{\tan }=$ $45 \mathrm{~km} \mathrm{~s}^{-1}$ ) seemingly contradicts this, suggesting that it is of comparable age to older field $\mathrm{T}$ dwarfs.

\subsubsection{MASS 0729-39 (T8pec)}

This object is one of four spectroscopically classified T8 dwarfs, the latest spectral type for T dwarfs currently known. While the peculiarities of 2MASS 0729-39 are not as evident as those for 2MASS $1324+63$, they are certainly noticeable and worth further exploration here. This source is peculiar because it has some excess flux in the $Y$-band peak, while the $J$-band peak, the $\mathrm{H}_{2} \mathrm{O}$ and $\mathrm{CH}_{4}$ absorption from $\sim 1.1$ to $1.2 \mu \mathrm{m}$, and the $\mathrm{H}_{2} \mathrm{O}$ absorption from 1.3 to $1.5 \mu \mathrm{m}$ match well to the T8 standard. There is also reduced flux in the $H$-band and $K$-band peaks compared to the $\mathrm{T} 8$ standard.

These peculiarities are indicative of a higher pressure photosphere, increasing $\mathrm{K}_{\mathrm{I}}$ wing absorption (causing slightly increased $Y$-band flux) and $\mathrm{CIA}_{2}$ absorption (causing depressed $H$ - and $K$-band flux). Such features were previously noted for the high surface gravity and metal-poor dwarf 2MASS 0937+29 (See

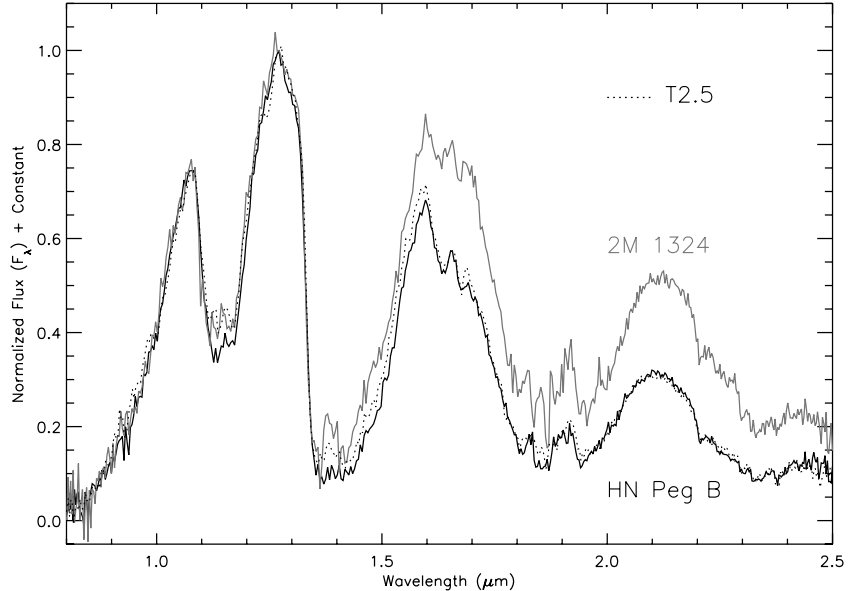

FIG. 8.-2MASS 1324+63 (T2.5:pec, solid gray line) overplotted in comparison to HN Peg B (T2.5, solid black line; Luhman et al. 2007), an 300 Myr old T dwarf. For comparison, a spectrum of a T2.5 dwarf constructed from the $\mathrm{T} 2$ and T3 spectral standards is also overplotted (dotted line). All spectra have been normalized at $1.27 \mu \mathrm{m}$.

Fig. 2 of Burgasser et al. 2006a). 2MASS 0729-39 may be a similarly relatively old and/or slightly metal-poor T dwarf.

\subsection{Temperatures and Gravities}

To examine the properties of our latest type $\mathrm{T}$ dwarfs in further detail, we made use of the semiempirical spectral index technique of Burgasser et al. (2006a) to estimate $T_{\text {eff }}$ and $\log g$. In brief, this method involves the comparison of $\mathrm{H}_{2} \mathrm{O}$ and color spectral ratios measured on the spectrum of a late-type $\mathrm{T}$ dwarf to the same ratios measured on theoretical condensate-free spectral models from Allard et al. (2001) and Burrows et al. (2006). The latter are calibrated to reproduce the measured indices for the NIR SpeX prism spectrum of G1 570D (Burgasser et al. $2000 \mathrm{a}$ ), which has parameters of $T_{\text {eff }}=782-821 \mathrm{~K}, \log g=$ 4.95-5.23, and $[\mathrm{Fe} / \mathrm{H}]=0.09 \pm 0.04$, based on empirical measurements and evolutionary models (Geballe et al. 2001; Saumon et al. 2006). The $\mathrm{H}_{2} \mathrm{O}$ and color ratios are separately sensitive to $T_{\text {eff }}$ and $\log g$ (for a given metallicity), and thus break the degeneracy between these parameters, which can then be used to infer mass and age with evolutionary models. As condensatefree spectral models generally provide poor fits for $T_{\text {eff }} \gtrsim 1200 \mathrm{~K}$, our analysis is generally confined to sources $\gtrsim \mathrm{T} 5$ (Golimowski et al. 2004). See Burgasser (2007) and Liebert \& Burgasser (2007) for examples of this spectral technique in application.

We were able to derive constraints for $T_{\text {eff }}$ and $\log g$ for five of the new T dwarfs in our sample using the $\mathrm{H}_{2} \mathrm{O}-J$ and $K / H$ ratios (the latter defined in Burgasser et al. 2006a). Figure 9 illustrates the fits for these sources, and Table 8 lists their estimated $T_{\text {eff }}$ and $\log g$ ranges assuming an uncertainty of $10 \%$ in the spectral ratio measurement. Note that additional systematic uncertainties of order $50 \mathrm{~K}$ and 0.2 dex should be included when interpreting these values. The $T_{\text {eff }}$ values are consistent with trends from parallax samples (Golimowski et al. 2004; Vrba et al. 2004), and show the expected decline in $T_{\text {eff }}$ with later spectral type. Note that the peculiar T8 2MASS 0729-39 has a relatively large surface gravity, consistent with our interpretation of this source in $\S$ 3.3.2. 2MASS $1007-45$, on the other hand, has a relatively low surface gravity, which is consistent with its brighter $K$-band flux peak as seen in Figure 3 relative to the T5 standard. Subsolar and supersolar metallicities can also reproduce these effects, respectively (Burgasser et al. 2006a, 2007; Liu et al. 2007; Saumon et al. 2007). 

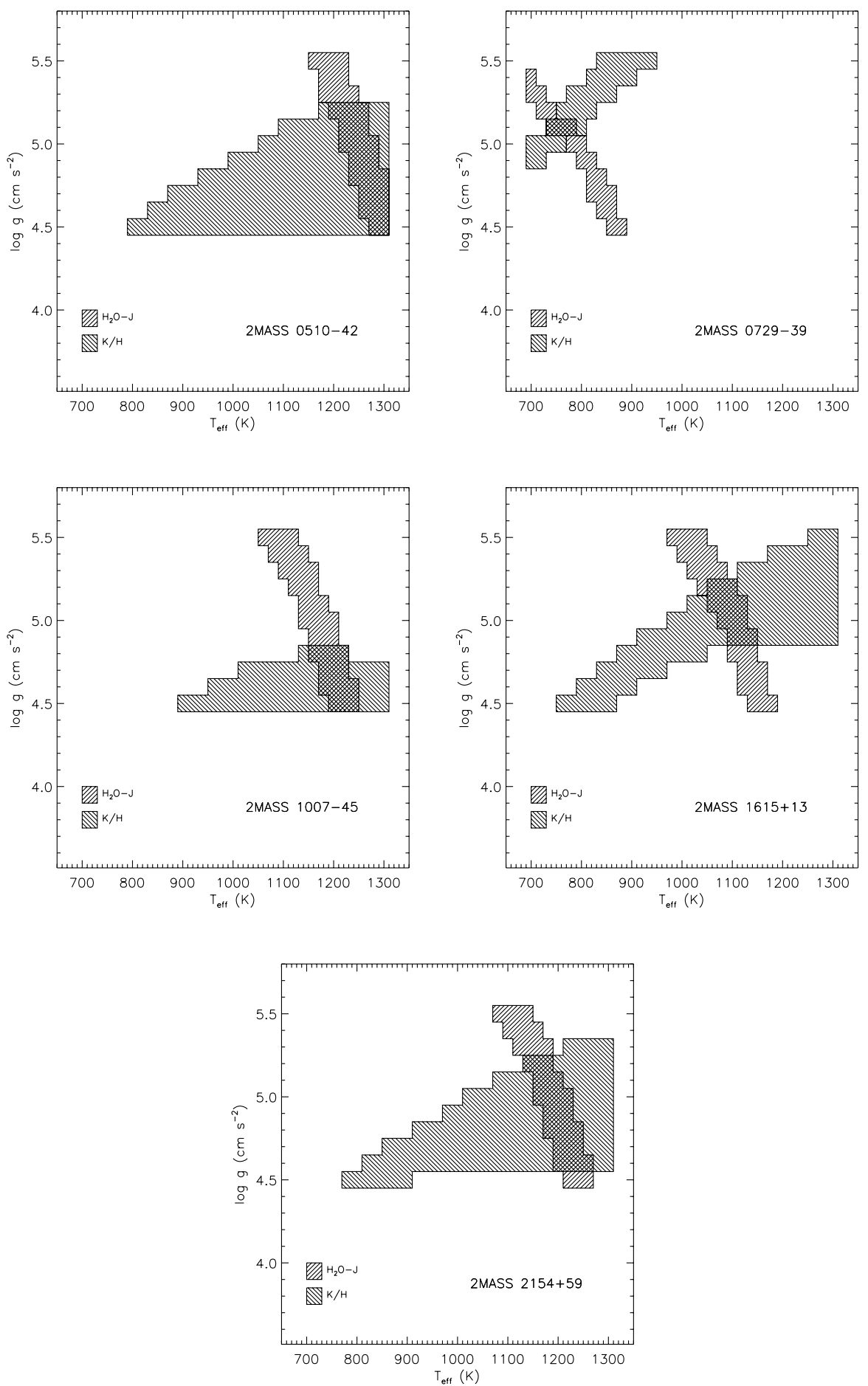

Fig. 9.- Spectral ratio measurements of $\mathrm{H}_{2} \mathrm{O}$ and $\mathrm{K} / \mathrm{H}$ (hatched regions) for 2MASS 0510-42 (T5), 2MASS 0729-39 (T8pec), 2MASS 1007-45 (T5), 2MASS $1615+13$ (T6), and 2MASS 2154+59 (T5) shown in $T_{\text {eff }}$ vs. $\log g$ phase space. Overlapping regions are the best fits; we list these values in Table 8.

TABLE 8

$T_{\text {eff And }} \log g$ Estimates for Late-Type T Dwarfs

\begin{tabular}{|c|c|c|c|c|c|}
\hline Object & Spectral Type & $\begin{array}{l}T_{\text {eff }} \\
(\mathrm{K})\end{array}$ & $\begin{array}{l}\log g \\
(\operatorname{cgs})\end{array}$ & $\begin{array}{l}\text { Mass } \\
\left(M_{\odot}\right)\end{array}$ & $\begin{array}{l}\text { Age } \\
\text { (Gyr) }\end{array}$ \\
\hline 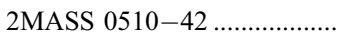 & T5 & $1200-1300$ & $4.5-5.2$ & $0.017-0.049$ & $0.13-2.1$ \\
\hline 2MASS $0729-39$...................... & T8pec & $740-780$ & 5.1 & $0.038-0.039$ & $3.8-4.1$ \\
\hline 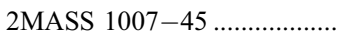 & T5 & $1160-1240$ & $4.5-4.8$ & $0.017-0.027$ & $0.2-0.4$ \\
\hline 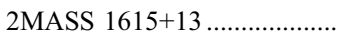 & $\mathrm{T} 6$ & $1060-1140$ & $4.9-5.2$ & $0.030-0.048$ & $0.6-2.9$ \\
\hline 2MASS $2154+59 \ldots \ldots \ldots$ & T5 & $1140-1240$ & $4.7-5.2$ & $0.023-0.048$ & $0.3-2.5$ \\
\hline
\end{tabular}


TABLE 9

T Dwarfs in the Solar Neighborhood (within 25 pc)

\begin{tabular}{|c|c|c|c|c|c|c|c|c|c|c|}
\hline \multirow[b]{2}{*}{ NAME OF T DWARF ${ }^{a}$} & \multirow{2}{*}{$\begin{array}{l}\text { Discovery } \\
\text { REFERENCE }\end{array}$} & \multirow{2}{*}{$\begin{array}{c}\text { NIR SPECTRAL } \\
\text { TyPE }\end{array}$} & \multirow{2}{*}{$\begin{array}{c}J^{\mathrm{b}} \\
(\mathrm{mag})\end{array}$} & \multirow{2}{*}{$\begin{array}{c}K_{s}^{\mathrm{b}} \\
(\mathrm{mag})\end{array}$} & \multicolumn{3}{|c|}{ Distance $(\mathrm{pc})$} & \multirow{2}{*}{$\begin{array}{l}\text { High-Res } \\
\text { IMAGING? }\end{array}$} & \multirow{2}{*}{$\begin{array}{l}\text { FOLLOW-UP } \\
\text { REFERENCE }\end{array}$} & \multirow[b]{2}{*}{ REMARKS } \\
\hline & & & & & From $J$ & From $\pi_{\text {trig }}$ & Adopted $^{\mathrm{c}}$ & & & \\
\hline \multicolumn{11}{|c|}{$d \leq 10 \mathrm{pc}$} \\
\hline$\epsilon$ Indi Bab (2MASS J2204-5646)..... & 1 & $\mathrm{~T} 1.0 / \mathrm{T} 6.0$ & 12.29 & 11.35 & 4.45 & 3.63 & 3.6 & Yes & 28 & Binary: $a=0.732^{\prime \prime}$ \\
\hline 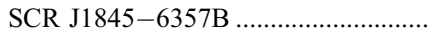 & 2 & T5.5 & $13.16^{\mathrm{d}}$ & $\ldots$ & $\ldots$ & 3.85 & 3.9 & Yes & 2 & Single \\
\hline 2MASSI J0415195-093506 .............. & 3 & $\mathrm{~T} 8.0$ & 15.70 & 15.43 & 7.58 & 5.74 & 5.7 & Yes & 29 & Single \\
\hline 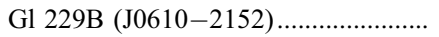 & 4 & $\mathrm{~T} 7.0 \mathrm{p}$ & 14.20 & 14.30 & 5.53 & 5.77 & 5.8 & Yes & 4,30 & Single \\
\hline G1 570D (2MASS J1457-2121)....... & 5 & $\mathrm{~T} 7.5$ & 15.32 & 15.24 & 7.73 & 5.91 & 5.9 & Yes & 31 & Single \\
\hline 2MASSI J0937347+293142 .............. & 3 & T6.0p & 14.65 & 15.27 & 9.32 & 6.14 & 6.1 & Yes & 31 & Single \\
\hline IPMS J013656.57+093347.3 ............. & 6 & $\mathrm{~T} 2.5$ & 13.46 & 12.56 & 8.27 & $\ldots$ & 8.3 & No & $\ldots$ & Single \\
\hline 2MASS J07290002-3954043* ......... & 7 & $\mathrm{~T} 8.0 \mathrm{p}$ & 15.92 & $\ldots$ & 8.41 & $\ldots$ & 8.4 & No & $\ldots$ & $\ldots$ \\
\hline 2MASS J15031961+2525196............ & 8 & T5.0 & 13.94 & 13.96 & 8.49 & $\ldots$ & 8.5 & Yes & 29 & Single \\
\hline 2MASS J09393548-2448279 ............ & 9 & $\mathrm{~T} 8.0$ & 15.98 & $\ldots$ & 8.65 & $\ldots$ & 8.7 & No & $\ldots$ & $\ldots$ \\
\hline 2MASSI J0727182+171001 .............. & 3 & $\mathrm{~T} 7.0$ & 15.60 & 15.56 & 10.53 & 9.08 & 9.1 & No & $\ldots$ & $\ldots$ \\
\hline 2MASS J03480772-6022270 ........... & 10 & $\mathrm{~T} 7.0$ & 15.32 & 15.60 & 9.25 & $\ldots$ & 9.3 & Yes & 29 & Single \\
\hline 2MASS J11145133-2618235 ............ & 9 & $\mathrm{~T} 7.5$ & 15.86 & $\ldots$ & 9.89 & $\cdots$ & 9.9 & No & $\ldots$ & $\ldots$ \\
\hline
\end{tabular}

$10 \mathrm{pc}<d<25 \mathrm{pc}$

\begin{tabular}{|c|c|c|c|c|c|c|c|c|c|c|}
\hline 2MASS J05591914-1404488 ............ & 11 & $\mathrm{~T} 4.5$ & 13.80 & 13.58 & 8.66 & 10.24 & 10.2 & Yes & 31 & Single \\
\hline 2MASS J12373919+6526148............ & 12 & T6.5 & 16.05 & $\ldots$ & 15.33 & 10.41 & 10.4 & Yes & 31 & Single \\
\hline 2MASSI J1047538+212423 ................ & 12 & T6.5 & 15.82 & $\ldots$ & 13.76 & 10.56 & 10.6 & Yes & 31 & Single \\
\hline 2MASSI J0243137-245329 .............. & 3 & T6.0 & 15.38 & 15.22 & 13.06 & 10.68 & 10.7 & Yes & 29 & Single \\
\hline SDSSp J162414.37+002915.6........... & 13 & T6.0 & 15.49 & $\ldots$ & 13.76 & 11.00 & 11.0 & Yes & 29 & Single \\
\hline 2MASSI J1217110-031113 .............. & 12 & $\mathrm{~T} 7.5$ & 15.86 & $\ldots$ & 9.90 & 11.01 & 11.0 & Yes & 29,27 & Single \\
\hline 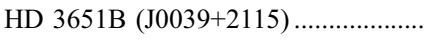 & 14 & $\mathrm{~T} 8$ & $16.16^{\mathrm{d}}$ & $16.87^{\mathrm{d}}$ & 9.39 & 11.11 & 11.1 & No & $\ldots$ & $\ldots$ \\
\hline 2MASSI J1546291-332511_............. & 3 & T5.5 & 15.63 & 15.49 & 16.66 & 11.36 & 11.4 & Yes & 31 & Single \\
\hline 2MASSI J1553022+153236AB.......... & 3 & $\mathrm{~T} 7.0$ & 15.83 & 15.51 & 11.68 & $\ldots$ & $>11.7$ & Yes & 29 & Binary: $a=0.349^{\prime \prime}$ \\
\hline SDSSp J125453.90-012247.4 .......... & 15 & $\mathrm{~T} 2.0$ & 14.89 & 13.84 & 15.76 & 11.78 & 11.8 & Yes & 29 & Single \\
\hline 2MASS J00345157+0523050 ............ & 16 & T6.5 & 15.54 & $\ldots$ & 12.08 & $\ldots$ & 12.1 & No & $\ldots$ & $\ldots$ \\
\hline 2MASS J00501994-3322402 ........... & 9 & $\mathrm{~T} 7.0$ & 15.93 & 15.24 & 12.24 & $\ldots$ & 12.2 & No & $\ldots$ & $\ldots$ \\
\hline 2MASS J12255432-2739466AB ...... & 12 & T6.0 & 15.26 & 15.07 & 12.35 & 13.32 & 13.3 & Yes & 31 & Binary: $a=0.282^{\prime \prime}$ \\
\hline 2MASS J18283572-4849046........... & 16 & $\mathrm{~T} 5.5$ & 15.18 & 15.18 & 13.50 & $\ldots$ & 13.5 & No & $\ldots$ & $\ldots$ \\
\hline 2MASSI J1534498-295227AB......... & 3 & T5.5 & 14.90 & 14.84 & 11.90 & 13.59 & 13.6 & Yes & 31 & Binary: $a=0.65^{\prime \prime}$ \\
\hline 2MASSI J2356547-155310 .............. & 3 & T5.5 & 15.82 & 15.77 & 18.21 & 14.50 & 14.5 & Yes & 31 & Single \\
\hline SDSSp J134646.45-003150.4 .......... & 17 & T6.5 & 16.00 & 15.77 & 14.96 & 14.64 & 14.6 & No & $\ldots$ & $\ldots$ \\
\hline 2MASS J22282889-4310262 ........... & 10 & T6.0 & 15.66 & 15.30 & 14.86 & $\ldots$ & 14.9 & Yes & 29 & Single \\
\hline SDSSp J042348.57-041403.5AB ..... & 18 & T0.0 & 14.47 & 12.93 & 11.10 & 15.17 & 15.2 & Yes & 29 & Binary: $a=0.164^{\prime \prime}$ \\
\hline SDSS J000013.54+255418.6 ............. & 19 & $\mathrm{~T} 4.5$ & 15.06 & 14.84 & 15.48 & $\ldots$ & 15.5 & No & $\ldots$ & $\ldots$ \\
\hline 2MASS J11061197+2754225* .......... & 7 & $\mathrm{~T} 2.5$ & 14.82 & 13.80 & 15.51 & $\ldots$ & 15.5 & No & $\ldots$ & $\ldots$ \\
\hline SDSS J162838.77+230821.1 …......... & 20 & $\mathrm{~T} 7.0$ & 16.46 & 15.87 & 15.63 & $\ldots$ & 15.6 & No & $\ldots$ & $\ldots$ \\
\hline SDSS J175805.46+463311.9 ............... & 19 & T6.5 & 16.15 & 15.47 & 16.05 & $\cdots$ & 16.1 & No & $\cdots$ & $\cdots$ \\
\hline SDSS J075840.33+324723.4 ............ & 19 & $\mathrm{~T} 2.0$ & 14.95 & 13.88 & 16.17 & $\ldots$ & 16.2 & No & $\ldots$ & $\ldots$ \\
\hline 2MASS J12314753+0847331............ & 16 & T5.5 & 15.57 & 15.22 & 16.20 & $\ldots$ & 16.2 & No & $\ldots$ & $\ldots$ \\
\hline 2MASS J11220826-3512363 ............ & 9 & $\mathrm{~T} 2.0$ & 15.02 & 14.38 & 16.72 & $\ldots$ & 16.7 & No & $\ldots$ & $\ldots$ \\
\hline 2MASSI J2254188+312349 ............... & 3 & $\mathrm{~T} 4.0$ & 15.26 & 14.90 & 18.01 & $\ldots$ & 18.0 & Yes & 29 & Single \\
\hline 2MASS J21392676+0220226............. & 21 & $\mathrm{~T} 1.5$ & 15.26 & 13.58 & 18.18 & $\cdots$ & 18.2 & No & $\cdots$ & $\ldots$ \\
\hline SDSS J152039.82+354619.8 .............. & 20 & T0.0 & 15.54 & 14.00 & 18.21 & $\ldots$ & 18.2 & No & $\ldots$ & $\ldots$ \\
\hline HN Peg B (J2144+1446) ....................... & 22 & $\mathrm{~T} 2.5$ & $15.86^{\mathrm{e}}$ & $15.12^{\mathrm{e}}$ & 25.03 & 18.39 & 18.4 & & & \\
\hline SDSS J120747.17+024424.8 ............. & 23 & T0.0 & 15.58 & 13.99 & 18.54 & $\ldots$ & 18.5 & No & $\ldots$ & $\ldots$ \\
\hline 2MASS J10073369-4555147* ......... & 7 & T5.0 & 15.65 & 15.56 & 18.68 & $\ldots$ & 18.7 & No & $\ldots$ & $\ldots$ \\
\hline 2MASS J23312378-4718274........... & 16 & $\mathrm{~T} 5.0$ & 15.66 & 15.39 & 18.76 & $\ldots$ & 18.8 & No & $\ldots$ & $\ldots$ \\
\hline 2MASS J21543318+5942187* ........... & 7 & T5.0 & 15.66 & $\ldots$ & 18.78 & $\cdots$ & 18.8 & No & $\cdots$ & $\ldots$ \\
\hline 2MASSW J0920122+351742AB ....... & 24 & T0.0 & 15.63 & 13.98 & 18.93 & $\ldots$ & $>18.9$ & Yes & 32 & Binary: $a=0.07^{\prime \prime}$ \\
\hline 2MASS J06020638+4043588* ........... & 7 & $\mathrm{~T} 4.5$ & 15.54 & 15.12 & 19.32 & $\ldots$ & 19.3 & No & $\ldots$ & $\ldots$ \\
\hline 2MASSI J0755480+221218 ............... & 3 & T5.0 & 15.728 & 15.75 & 19.36 & $\cdots$ & 19.4 & Yes & 29 & Single \\
\hline 2MASS J05160945-0445499............ & 10 & T5.5 & 15.98 & 15.49 & 19.60 & $\ldots$ & 19.6 & Yes & 29 & Single \\
\hline SDSS J150411.63+102718.4 .............. & 20 & $\mathrm{~T} 7.0$ & 17.03 & 17.02 & 20.36 & $\ldots$ & 20.4 & No & $\ldots$ & $\ldots$ \\
\hline 2MASS J16150410+1340070* ........... & 7 & T6.0 & 16.35 & $\ldots$ & 20.40 & $\ldots$ & 20.4 & No & $\ldots$ & $\ldots$ \\
\hline G1 337CD (2MASS J0912+1459)...... & 25 & T0.0 & 15.51 & 14.04 & 17.97 & 20.48 & 20.5 & Yes & 33 & Binary: $a=0.53^{\prime \prime}$ \\
\hline 2MASS J19010601+4718136............. & 16 & $\mathrm{~T} 5.0$ & 15.86 & 15.64 & 20.54 & $\ldots$ & 20.5 & No & $\ldots$ & $\ldots$ \\
\hline SDSS J015141.69+124429.6 ............. & 18 & $\mathrm{~T} 1.0$ & 16.57 & 15.18 & 31.89 & 21.40 & 21.4 & Yes & 29 & Single \\
\hline 2MASS J13243559+6358284* .......... & 7 & $\mathrm{~T} 2.0 \mathrm{p}$ & 15.60 & 14.06 & 21.80 & $\ldots$ & 21.8 & No & $\ldots$ & $\ldots$ \\
\hline 2MASS J14044941-3159329* ......... & 7 & $\mathrm{~T} 2.5$ & 15.58 & 14.54 & 21.97 & $\ldots$ & 22.0 & No & $\ldots$ & $\ldots$ \\
\hline SDSS J032553.17+042540.1 ............. & 20 & T5.5 & 16.25 & 16.53 & 22.20 & $\ldots$ & 22.2 & No & $\ldots$ & $\ldots$ \\
\hline
\end{tabular}


TABLE 9-Continued

\begin{tabular}{|c|c|c|c|c|c|c|c|c|c|c|}
\hline \multirow[b]{2}{*}{ NAME OF T DwARF ${ }^{a}$} & \multirow{2}{*}{$\begin{array}{l}\text { Discovery } \\
\text { REFERENCE }\end{array}$} & \multirow{2}{*}{$\begin{array}{c}\text { NIR SPECTRAL } \\
\text { TYPE }\end{array}$} & \multirow{2}{*}{$\begin{array}{c}J^{\mathrm{b}} \\
(\mathrm{mag})\end{array}$} & \multirow{2}{*}{$\begin{array}{c}K_{s}^{\mathrm{b}} \\
(\mathrm{mag})\end{array}$} & \multicolumn{3}{|c|}{ Distance $(\mathrm{pc})$} & \multirow{2}{*}{$\begin{array}{l}\text { High-Res } \\
\text { IMAGING? }\end{array}$} & \multirow{2}{*}{$\begin{array}{l}\text { FOLLOW-UP } \\
\text { REFERENCE }\end{array}$} & \multirow[b]{2}{*}{ Remarks } \\
\hline & & & & & From $J$ & From $\pi_{\text {trig }}$ & Adopted $^{\mathrm{c}}$ & & & \\
\hline SDSS J212413.89+010000.3 …............... & 19 & T5.0 & 16.03 & 16.14 & 22.26 & $\ldots$ & 22.3 & No & $\ldots$ & $\ldots$ \\
\hline 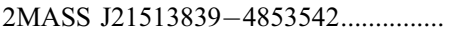 & 26 & T4.0 & 15.73 & 15.43 & 22.34 & $\ldots$ & 22.3 & No & $\ldots$ & $\ldots$ \\
\hline 2MASS J04070885+1514565 .................. & 16 & $\mathrm{~T} 5.0$ & 16.06 & 15.92 & 22.51 & $\ldots$ & 22.5 & No & $\ldots$ & $\ldots$ \\
\hline SDSS J151114.66+060742.9 …............... & 20 & T0.0 & 16.02 & 14.54 & 22.67 & $\ldots$ & 22.7 & No & $\ldots$ & $\ldots$ \\
\hline SDSSp J092615.38+584720.9AB ......... & 18 & $\mathrm{~T} 4.5$ & 15.90 & 15.45 & 22.73 & $\ldots$ & $>22.7$ & Yes & 29 & Binary: $a=0.070^{\prime \prime}$ \\
\hline SDSS J105213.51+442255.7 ................... & 20 & T0.5 & 15.96 & 14.57 & 23.08 & $\ldots$ & 23.1 & No & $\ldots$ & $\ldots$ \\
\hline SDSSp J111010.01+011613.1 .................. & 18 & T5.5 & 16.34 & 15.13 & 23.12 & $\ldots$ & 23.1 & Yes & 29 & Single \\
\hline SDSS J074149.15+235127.5 ….............. & 19 & T5.0 & 16.16 & 15.85 & 23.61 & $\ldots$ & 23.6 & No & $\ldots$ & $\ldots$ \\
\hline SDSS J163022.92+081822.0 ................... & 20 & T5.5 & 16.40 & 16.61 & 23.69 & $\ldots$ & 23.7 & No & $\ldots$ & $\ldots$ \\
\hline SDSS J074201.41+205520.5 _................... & 19 & $\mathrm{~T} 5.0$ & 16.19 & 15.23 & 23.99 & $\ldots$ & 24.0 & No & $\ldots$ & $\ldots$ \\
\hline 2MASS J05103520-4208140* ................ & 7 & $\mathrm{~T} 5.0$ & 16.22 & 16.00 & 24.31 & $\ldots$ & 24.3 & No & $\ldots$ & $\ldots$ \\
\hline 2MASS J05185995-2828372AB .......... & 27 & $\mathrm{~T} 1.0$ & 15.98 & 14.16 & 24.32 & $\ldots$ & $>24.3$ & Yes & 29 & Binary: $a=0.051^{\prime \prime}$ \\
\hline 2MASSI J2339101+135230 …….............. & 3 & T5.0 & 16.24 & 16.15 & 24.50 & $\ldots$ & 24.5 & Yes & 29 & Single \\
\hline
\end{tabular}

${ }^{\text {a }}$ Asterisks mark new additions reported in this paper.

${ }^{b}$ Photometry from 2MASS All-Sky Point Source Catalog.

${ }^{c}$ Distance adopted from trigonometric parallax if measured.

${ }^{\mathrm{d}} H$-band magnitude from Biller et al. (2006).

e Photometry is from Luhman et al. (2007).

References.-(1) Scholz et al. 2003; (2) Biller et al. 2006; (3) Burgasser et al. 2002; (4) Nakajima et al. 1995; (5) Burgasser et al. 2000a; (6) Artigau et al. 2006; (7) this paper; (8) Burgasser et al. 2003a; (9) Tinney et al. 2005; (10) Burgasser et al. 2003c; (11) Burgasser et al. 2000b; (12) Burgasser et al. 1999; (13) Straus et al. 1999; (14) Mugrauer et al. 2006; (15) Leggett et al. 2000; (16) Burgasser et al. 2004b; (17) Tsvetanov et al. 2000; (18) Geballe et al. 2002; (19) Knapp et al. 2004; (20) Chiu et al. 2006; (21) K. L. Cruz et al. 2007, in preparation; (22) Luhman et al. 2007; (23) Hawley et al. 2002; (24) Kirkpatrick et al. 2000; (25) Wilson et al. 2001; (26) Ellis et al. 2005; (27) Cruz et al. 2004; (28) McCaughrean et al. 2004; (29) Burgasser et al. 2006c; (30) Golimowski et al. 1998; (31) Burgasser et al. 2003b; (32) Reid et al. 2001; (33) Burgasser et al. 2005a.

Using the solar-metallicity evolutionary models of Burrows et al. (2001) we estimated masses and ages for these five sources; ranges (without including systematic uncertainties) are given in Table 8. As expected, low (high) surface gravities result in low (high) mass and age estimates. Since metallicity effects can mimic surface gravity effects on the $K$ band (in both cases modulating the relative opacity of $\mathrm{H}_{2}$ ), care should be taken in interpreting these values.

\section{DISCUSSION: THE SOLAR NEIGHBORHOOD}

To place our discoveries in context and to review the current state of high-resolution imaging for the nearest $\mathrm{T}$ dwarfs, we have constructed an up-to-date census of $\mathrm{T}$ dwarfs in the solar neighborhood $(<25 \mathrm{pc})$. This list was constructed from the L \& T dwarf compendium maintained by Kirkpatrick, Gelino, \& Burgasser. ${ }^{14}$ We computed spectrophotometric distance estimates for the entire list of T dwarfs, using the $M_{J}$ versus NIR SpT relation derived by Liu et al. (2006) (excluding known binaries). Those $\mathrm{T}$ dwarfs with trigonometric parallaxes have their distance estimates superseded by these measurements. For T dwarfs known to be binaries but without parallaxes, we have placed lower limits on their spectrophotometric distance estimate. Within $25 \mathrm{pc}$, this list includes a total of $72 \mathrm{~T}$ dwarfs, including nine new additions reported here (see Table 9). These new additions represent an $\sim 14 \%$ increase in the $25 \mathrm{pc}$ census of T dwarfs. Only one T dwarf from the compendium meeting these criteria was excluded from this list: 2MASS J11263991-5003550 (NIR T0; Folkes et al. 2007), which A. J. Burgasser et al. (2007, in preparation) demonstrate has a mid-type-L dwarf optical spectral morphology.

Within $10 \mathrm{pc}$, there are currently 14 known $\mathrm{T}$ dwarfs, one of which, 2MASS 0729-39 (the eighth closest T dwarf to the Sun at $8.4 \mathrm{pc}$ ), is reported here. Of these $14 \mathrm{~T}$ dwarfs, five are companions to more massive stars. To date, over half of these T dwarfs have been followed-up with high-resolution imaging, with one

\footnotetext{
${ }^{14}$ See http://dwarfarchives.org.
}

revealed as a tight binary, the nearest T dwarf, $\epsilon$ Indi Bab (Scholz et al. 2003; McCaughrean et al. 2004). This yields a binary fraction of $\sim 13 \%$ and a lower limit to the observed space density of $3 \times 10^{-3} \mathrm{~T}$ dwarfs pc ${ }^{-3}$ for the $10 \mathrm{pc}$ sample. S. Metchev et al. (2007, in preparation) performed Monte Carlo simulations, predicting $\sim 30 \mathrm{~T} 0-\mathrm{T} 8 \mathrm{dwarfs}$ within $10 \mathrm{pc}$ (a space density of $7_{-3.0}^{+3.2} \times 10^{-3} \mathrm{pc}^{-3}$ ), roughly twice the known population. The inputs for these simulations were based on a critical examination of T dwarf candidates chosen from a cross-correlation of SDSS and 2MASS, which helps to eliminate the 2MASS selection bias for early-T dwarfs and the SDSS selection bias for very late-T dwarfs. This density estimate exceeds that derived both for T5-T8 dwarfs $\left(4.2 \times 10^{-3} \mathrm{pc}^{-3}\right.$; Burgasser et al. 2002) and for L dwarfs $(3.8 \times$ $10^{-3} \mathrm{pc}^{-3}$; Cruz et al. 2007).

Between 10 and $25 \mathrm{pc}$, there are currently 59 known $\mathrm{T}$ dwarfs, eight of which we report here. One caveat to this set is that the distances we assigned to nonparallax sources are biased into this sample if they are binary (i.e., they are really farther away, potentially outside $25 \mathrm{pc}$ ). The state of high-resolution imaging for this sample is not nearly as complete as for $d<10 \mathrm{pc}$, with only 23 out of the 59 known T dwarfs having follow-up. Of these 23 $\mathrm{T}$ dwarfs, seven have been identified as binaries (Burgasser et al. $2006 \mathrm{c}$ and references therein), yielding a binary fraction of $\sim 30 \%$ for this sample. This entire list represents an interesting distance-limited subset of all known T dwarfs, complete followup (both high-resolution imaging and parallax measurements) of which could greatly bolster binarity statistics and lead to a more complete understanding of brown dwarf formation. Based on the number of known T dwarfs within $10 \mathrm{pc}$, there should be $\gtrsim 200 \mathrm{~T}$ dwarfs within $10 \mathrm{pc}<d<25 \mathrm{pc}$ (S. Metchev et al. [2007, in preparation] predict $\sim 430$ ), so the current census is highly incomplete.

We also note that, like the prototypes of the $\mathrm{L}$ and T spectral classes (GD 165B [Becklin \& Zuckerman 1988] and Gl 229B [Nakajima et al. 1995; Oppenheimer et al. 1995], respectively), which are both companions, the prototype of the $Y$ spectral class (hypothetical dwarfs cooler than type T; Kirkpatrick et al. 1999) could first be 
identified as a companion to one of these T dwarfs. This should be ample motivation for the brown dwarf community to continue high angular resolution imaging of our nearest cool neighbors.

\section{CONCLUSIONS}

We have reported on the discovery of 11 new $\mathrm{T}$ dwarfs found during the course of two surveys using the 2MASS database. Using low-resolution prism spectroscopy $(R \sim 150)$ on IRTF SpeX, we have classified these $T$ dwarfs in the near-infrared from T2 to T8. All of these T dwarfs are nearby, with spectrophotometric distance estimates of $\sim 8.4$ to $29.0 \mathrm{pc}$. One of these T dwarfs, 2MASS J13243559+6358284, we type as T2:pec and postulate that its spectroscopic peculiarities are the result of reduced collision induced absorption by $\mathrm{H}_{2}$, indicative of it being a young object ( $<300 \mathrm{Myr}$ ), or that it is an L/T transition binary. To provide models for suspected or known binaries, we constructed a suite of synthetic spectra and computed their $\mathrm{H}_{2} \mathrm{O}$ and $\mathrm{CH}_{4}$ indices. We have also reviewed the state of high-resolution follow-up of T dwarfs in the solar neighborhood with $d<25 \mathrm{pc}$ and make the case for completing follow-up of this sample to improve the poorly sampled binary statistics throughout the T spectral class for this distance-limited set.

D. L. L. thanks J. Rayner for advising her for part of this project and for a careful read of the manuscript. We would like to thank J. Kartaltepe and Y. Kakazu for imaging several of our targets with the UH 2.2 m ULBCAM and for teaching D. L. L. how to use the instrument. We thank S. Metchev for helping us with the SDSS Catalog, Mike Cushing for useful discussions, and Kevin Luhman for kindly providing the spectrum of HN Peg B. We would also like to thank our telescope operators on IRTF: D. Griep and B. Golisch. This paper uses data from the IRTF Spectral Library (http://irtfweb.ifa.hawaii.edu/ spex/spexlibrary/ IRTFlibrary.html) and from http://DwarfArchives.org. This publication also makes use of data products from the Two Micron All Sky Survey, which is a joint project of the University of Massachusetts and the Infrared Processing and Analysis Center/ California Institute of Technology, funded by the National Aeronautics and Space Administration and the National Science Foundation. D. L. L. was a guest user of the Canadian Astronomy Data Centre, which is operated by the Herzberg Institute of Astrophysics, National Research Council of Canada. This research has also made use of the NASA/IPAC Infrared Science Archive, which is operated by the Jet Propulsion Laboratory, California Institute of Technology, under contract with the National Aeronautics and Space Administration. As all spectroscopic and imaging follow-up data were obtained from the summit of Mauna Kea, the authors wish to recognize and acknowledge the very significant cultural role and reverence that this mountaintop has always had within the indigenous Hawaiian community. We are most fortunate to have the opportunity to conduct observations on the summit.

\section{REFERENCES}

Allard, F., Hauschildt, P. H., Alexander, D. R., Tamanai, A., \& Schweitzer, A. 2001, ApJ, 556, 357

Artigau, É., Doyon, R., Lafrenière, D., Nadeau, D., Robert, J., \& Albert, L. 2006, ApJ, 651, L57

Becklin, E. E., \& Zuckerman, B. 1988, Nature, 336, 656

Biller, B. A., Kasper, M., Close, L. M., Brandner, W., \& Kellner, S. 2006, ApJ, 641, L141

Borysow, A., Jorgensen, U. G., \& Zheng, C. 1997, A\&A, 324, 185

Burgasser, A. J. 2007, preprint (astro-ph/0701793)

Burgasser, A. J., Burrows, A., \& Kirkpatrick, J. D. 2006a, ApJ, 639, 1095

Burgasser, A. J., Geballe, T. R., Leggett, S. K., Kirkpatrick, J. D., \& Golimowski, D. A. 2006b, ApJ, 637, 1067

Burgasser, A. J., Kirkpatrick, J. D., Cruz, K. L., Reid, I. N., Leggett, S. K., Liebert, J., Burrows, A., \& Brown, M. E. 2006c, ApJS, 166, 585

Burgasser, A. J., Kirkpatrick, J. D., \& Lowrance, P. J. 2005a, AJ, 129, 2849

Burgasser, A. J., Kirkpatrick, J. D., McElwain, M. W., Cutri, R. M., Burgasser, A. J., \& Skrutskie, M. F. 2003a, AJ, 125, 850

Burgasser, A. J., Kirkpatrick, J. D., McGovern, M. R., McLean, I. S., Prato, L., \& Reid, I. N. 2004a, ApJ, 604, 827

Burgasser, A. J., Kirkpatrick, J. D., Reid, I. N., Brown, M. E., Miskey, C. L., \& Gizis, J. E. 2003b, ApJ, 586, 512

Burgasser, A. J., McElwain, M. W., \& Kirkpatrick, J. D. 2003c, AJ, 126, 2487

Burgasser, A. J., McElwain, M. W., Kirkpatrick, J. D., Cruz, K. L., Tinney, C. G., \& Reid, I. N. 2004b, AJ, 127, 2856

Burgasser, A. J., Reid, I. N., Leggett, S. K., Kirkpatrick, J. D., Liebert, J., \& Burrows, A. 2005b, ApJ, 634, L177

Burgasser, A. J., et al. 1999, ApJ, 522, L65

. 2000a, ApJ, 531, L57

2000 b, AJ, 120,1100

2002, ApJ, 564, 421

Burrows, A., Hubbard, W. B., Lunine, J. I., \& Liebert, J. 2001, Rev. Mod. Phys., 73, 719

Burrows, A., Sudarsky, D., \& Hubeny, I. 2006, ApJ, 640, 1063

Burrows, A., et al. 1997, ApJ, 491, 856

Chiu, K., Fan, X., Leggett, S. K., Golimowski, D. A., Zheng, W., Geballe, T. R., Schneider, D. P., \& Brinkmann, J. 2006, AJ, 131, 2722

Cruz, K. L., Burgasser, A. J., Reid, I. N., \& Liebert, J. 2004, ApJ, 604, L61

Cruz, K. L., et al. 2007, AJ, 133, 439

Cushing, M. C., Rayner, J. T., \& Vacca, W. D. 2005, ApJ, 623, 1115

Cushing, M. C., Vacca, W. D., \& Rayner, J. T. 2004, PASP, 116, 362

Ellis, S. C., Tinney, C. G., Burgasser, A. J., Kirkpatrick, J. D., \& McElwain, M. W. 2005, AJ, 130, 2347
Folkes, S. L., Pinfield, D. J., Kendall, T. R., \& Jones., H. R. A. 2007, MNRAS, 378,901

Geballe, T. R., Saumon, D., Leggett, S. K., Knapp, G. R., Marley, M. S., \& Lodders, K. 2001, ApJ, 556, 373

Geballe, T. R., et al. 2002, ApJ, 564, 466

Golimowski, D. A., Burrows, C. J., Kulkarni, S. R., Oppenheimer, B. R., \& Brukardt, R. A. 1998, AJ, 115, 2579

Golimowski, D. A., et al. 2004, AJ, 127, 3516

Grav, T., \& Holman, M. J. 2004, ApJ, 605, L141

Hawley, S. L., et al. 2002, AJ, 123, 3409

Hillenbrand, L. A., Foster, J. B., Persson, S. E., \& Matthews, K. 2002, PASP, 114,708

Kirkpatrick, J. D. 2005, ARA\&A, 43, 195

Kirkpatrick, J. D., Barman, T. S., Burgasser, A. J., McGovern, M. R., McLean, I. S., TInney, C. G., \& Lowrance, P. J. 2006, ApJ, 639, 1120

Kirkpatrick, J. D., et al. 1999, ApJ, 519, 802

2000, AJ, 120, 447

Knapp, G. R., et al. 2004, AJ, 127, 3553

Leggett, S. K., et al. 2000, ApJ, 536, L35

Liebert, J., \& Burgasser, A. J. 2007, ApJ, 655, 522

Liu, M. C., Leggett, S. K., \& Chiu, K. 2007, ApJ, 660, 1507

Liu, M. L., Leggett, S. K., Golimowski, D. A., Chiu, K., Fan, X., Gellae, T. R., Schneider, D. P., \& Brinkmann, J. 2006, ApJ, 647, 1393

Luhman, K. L., et al. 2007, ApJ, 654, 570

Martín, E. L., \& Osorio, M. R. Z. 2003, ApJ, 593, L113

McCaughrean, M. J., Close, L. M., Scholz, R.-D., Lenzen, R., Biller, B., Brandner, W., Hartung, M., \& Lodieu, N. 2004, A\&A, 413, 1029

Monet, D. G., et al. 1998, USNO-A2.0 Catalog (Washington: USNO)

Mugrauer, A., Seifahrt, R., \& Neuhaeuser, T. M. 2006, MNRAS, 373, L31

Nakajima, T., Oppenheimer, B. R., Kulkarni, S. R., Golimowski, D. A., Matthews, K., \& Durrance, S. T. 1995, Nature, 378, 463

Oppenheimer, B. R., Kulkarni, S. R., Matthews, K., \& Nakajima, T. 1995, Science, 270, 1478

Ramsay, S. K., Mountain, C. M., \& Geballe, T. R. 1992, MNRAS, 259, 751

Rayner, J. T., Toomey, D. W., Onaka, P. M., Denault, A. J., Stahlberger, W. E., Vacca, W. D., Cushing, M. C., \& Wang, S. 2003, PASP, 115, 362

Reid, I. N., Gizis, J. E., Kirkpatrick, J. D., \& Koerner, D. W. 2001, AJ, 121, 489

Reid, I. N., Lewitus, E., Burgasser, A. J., \& Cruz, K. L. 2006, ApJ, 639, 1114

Saumon, D., Bergeron, P., Lunine, J. I., Hubbard, W. B., \& Burrows, A. 1994, ApJ, 424, 333

Saumon, D., Marley, M. S., Cushing, M. C., Leggett, S. K., Roellig, T. L., Lodders, K., \& Freedman, R. S. 2006, ApJ, 647, 552 
Saumon, D., et al. 2007, ApJ, 656, 1136

Scholz, R.-D., McCaughrean, M., J., Lodieu, N., \& Kuhlbrodt, B. 2003, A\&A, 398, L29

Skrutskie, M. F., et al. 2006, AJ, 131, 1163

Straus, M. A., et al. 1999, ApJ, 522, L61

Stumpf, M. B., Brandner, W., \& Henning, T. 2005, in Protostars and Planets V, ed. B. Reipurth, D. Jewitt, \& K. Keil (Tucson: Univ. Arizona Press), 8571

Tinney, C. G., Burgasser, A. J., Kirkpatrick, J. D., \& McElwain, M. W. 2005, AJ, 130, 2326
Tsvetanov, Z. I., et al. 2000, ApJ, 531, L61

Vacca, W. D., Cushing, M. C., \& Rayner, J. T. 2003, PASP, 115, 389

Vrba, F. J., Henden, A. A., Luginbuhl, C. B., Guetter, H. H., \& Munn, J. A. 2004, AJ, 127, 2948

Wilson, J. C., Kirkpatrick, J. D., Gizis, J. E., Skrutskie, M. F., Monet, D. G., \& Houch, J. R. 2001, AJ, 122, 1989

York, D. G., et al. 2000, AJ, 120, 1579

Zapatero Osorio, M. R., Béjar, V. J. S., Martín, E. L., Rebolo, R., Barrado y Navascués, D., Mundt, R., Eislöffel, J., \& Caballero, J. A. 2002, ApJ, 578, 536 